Interfaces and Free Boundaries 16 (2014), 105-136

DOI $10.4171 / \mathrm{IFB} / 315$

\title{
On the equations of landscape formation
}

\author{
Alex CHEN \\ Statistical and Applied Mathematical Sciences Institute; Department of Mathematics, UNC Chapel \\ Hill, NC 27599-3250, USA \\ E-mail: aschen@live.unc.edu \\ JÉRÔME DARBON \\ CMLA, ENS de Cachan, 61 avenue du président Wilson, 94235 Cachan, France \\ E-mail: jerome.darbon@cmla.ens-cachan.fr \\ GiUseppe ButTAZZO \\ Dipartimento di Matematica, Università di Pisa, Largo B. Pontecorvo, 5, 56127 Pisa, Italy \\ E-mail: buttazzo@dm.unipi.it \\ FilipPo SANTAMBRogio \\ Laboratoire de Mathématiques d'Orsay, Bâtiment 425, bureau 309, Université Paris-Sud, \\ 91405 Orsay cedex, France \\ E-mail: filippo.santambrogio@math.u-psud.fr \\ JEAN-MiCHEL MOREL \\ CMLA, ENS de Cachan, 61 avenue du président Wilson, 94235 Cachan, France \\ E-mail:morel@cmla.ens-cachan.fr
}

[Received 2 February 2013 and in revised form 24 November 2013]

This paper starts by establishing a list of the main physical and empirical laws that have been proposed for modeling landscape evolution. Then it proposes a detailed mathematical discussion of several classic landscape evolution models. This discussion permits to distinguish a priori laws from a posteriori laws governing landscape evolution. While the first kind of law relies on physical principles, the second kind stems from the observation of actual stable landscapes. It emerges from the analysis of several models that the a posteriori laws are often used to create hybrid models combining fundamental physical laws with empirical modeling. Such models can be valid near equilibrium. Nevertheless, this situation is not satisfactory from a mathematical standpoint. One would like to be able to deduce the a posteriori laws from a mathematical analysis, or at the very least to observe them from numerical simulations using only a priori physical laws. Such a mathematical and numerical research programme is sketched here. We propose a minimal landscape model coping with the main features of all models. This model singles out three spatially distributed scalar state variables, namely the landscape elevation, the water elevation, and the sediment concentration in water. These state variables are linked by three partial differential equations coping with the three main features identified in the literature as the main phenomena shaping a landscape: erosion, sedimentation and creep, and respecting conservation laws for water and sediment. Based on these equations, the first numerical simulations confirm that valley formation and drainage network formation can be simulated on virgin artificial landscapes. A first surprising result seems to emerge from these simulations: the valley spacing depends not only on a relation between the creep and erosion parameters, but is also strongly influenced by the sedimentation rate and by the rainfall intensity. The conjectured mathematical instability and non-uniqueness of landscape evolution is 


\begin{abstract}
illustrated numerically. On the other hand numerical stability of real landscape topographies under realistic values for their evolution is also observed. The overall modeling and numerical tests suggest that existence and partial regularity results might be obtained under minimal assumptions (such as a positive rain density or a positive creep model).
\end{abstract}

2010 Mathematics Subject Classification: Primary 8602; Secondary 8608.

Keywords: Synthetic landscape evolution, Physical model, Partial differential equations, River networks, Conservation laws, Stream incision

\title{
1. Introduction
}

The problem of the laws of landscape evolution was raised by Gilbert and Dutton [18] in 1877. The main laws were formulated by Gilbert after a clever qualitative analysis of many real landscape observations and photographs [17] in 1909. In 1892, Davis [10] had already proposed an explanation of the convexity of hilltops, ascribing it to "creep." There is a long tradition of studying landscape evolution with a very large toolbox of laws and relevant parameters. The classic Horton (1945) article [22] on the erosional development of streams and their drainage basins starts with a list of not less than 57 different symbols referring to state variables and observable parameters in a landscape. Another classic paper [70] has 30 and [60] has not less than 70. The first mentioned paper develops the ambition of identifying universal scaling laws for river networks and the second attempts a model for coupling channel network growth and hillslope evolution. An entire book [52] is dedicated to many discrete landscape models and observations, with particular attention to the fractal nature of river networks and their scaling laws. There is indeed a list of empirical scaling laws in landscape morphology, such as Hack's Law $L=C A^{h}, h \approx 0.6$, an empirical relationship between the length of streams and the area of their basins. Nevertheless, the derivation of such scaling laws from first principles is still a wide open problem.

A striking feature of most papers on landscape modeling is their modeling prudence. Each law is stated separately and often under several variants in the same paper. This is the case (e.g.) for $[11,54,55,65]$, where the equations which we will discuss here are nonetheless present.

Prudence is necessary even when statistical observations are strikingly consistent with a certain model. This consistency is often limited by the authors to a certain class of landscapes [23], and does not prove the generality of the model. For instance in [47] the study of characteristic valley spacings is limited to five sites where "erosion and transport are dominated by stream channel incision and by diffusive soil creep." These authors add that "Competition between soil creep and stream incision is the primary mechanism that controls valley spacing in these landscapes."

Even more prudence is observed in the evaluation of the influence of the time scale of landscape evolution. As pointed out in [21], the actual state of a landscape may be transitional and may reflect the effects of erosion and transport phenomena on different time scales. According to these authors "the channels of the Dragon's Back pressure ridge respond to changes in rock uplift rates over thousands of years, whereas hillslope processes may take more than an order of magnitude longer to adjust to changes in rock uplift rates." Dynamic modeling was also addressed in [50], and shows that in a periodic climate the form of the landscape cannot be explained by a static model. Likewise, as pointed out in [47] soil quality parameters are difficult to measure directly because "erosion is usually slow or episodic, and because present-day rates may not be representative of long-term rates." 
Tucker and Bras [65] summarized the valley formation modeling dilemma, whether it can be modeled by a steady model or by periodic events: "Valleys form where flow convergence causes rill or gully excavation by runoff erosion to outpace infilling by diffusive processes such as rainsplash." Yet, "an alternative view is that valley and channel formation are controlled by geomorphic thresholds. A number of researchers have argued, for example, that hillslope-valley transitions occur where a threshold for runoff erosion is regularly exceeded during large storms." This view was supported by Horton [22], Montgomery and Dietrich [42], Willgoose et al. [70]. Another modeling issue is the dilemma between transport-limited with regolith versus the detachment-limited case for hard bedrock [65]: "In a landscape mantled by easily-detachable regolith the water-borne sediment flux $Q_{s}$ is equal to the total sediment transport capacity (referred to as the transport-limited case), whereas if the surface materials are resistant to detachment (e.g., bedrock, coarse or cohesive sediment, or a thickly vegetated surface), $Q_{s}$ will generally be less than the sediment transport capacity (the detachment-limited case)."

Nevertheless, Izumi and Parker [29] consider that transitional solutions of the water runoff equations can be neglected, the flow being assumed steady during the erosion process: "In accordance with the usual quasi-steady assumption of erodible-bed flow problems, it is assumed that the setup time upon commencement of rainfall to achieve a steady flow is small compared to the time required for significant erosion to occur. The flow equations are thus approximated as steady."

The mathematical modeling method of landscape evolution was already under consideration in Gilbert [17], where qualitative mathematical arguments are well present. Quantitative laws go back at least to Culling (1960) [9]. Smith and Bretherton [60] also argue in favor of simplifying assumptions to get some kind of general intuition on landscape morphology.

The object of the present paper is fourfold. A first objective is to establish a list of the main physical laws that have been proposed for landscape evolution. Because of the many variants and of the dilemmas mentioned above, this list cannot be exhaustive, but we will attempt to go back to first physical principles. The second objective is to show that with this short list of laws one is able to understand and classify landscape models. A third objective is to deduce from this analysis a landscape model coping with the main features of all models. Here a decision will have to be made and justified. We shall opt whenever possible for an all-physical model and avoid using in the modeling empirical laws deduced from observations. Indeed, such empirical models restrain the validity of models, because they are valid for stabilized landscapes only. A fourth objective is to perform first experiments to see if the proposed model, a system of three partial differential equations, copes with the main features and phenomena expected from a landscape evolution. Among the striking features of landscape evolution that will be retrieved in numerical simulations are the formation of valleys, lakes and sedimentation fronts and a strong dependence on initial conditions. The proposed model is entirely derived from the simplified conservation laws already used in the literature and from the simplest versions of the erosion, sedimentation and creep laws. This leads to a system of three partial differential equations.

Our final objective is to motivate mathematicians to work on the proposed model, by raising some striking properties of landscape evolution models, such as their non-uniqueness and instability under the no-creep assumption, the suggestion that existence and partial regularity results might be obtained in spite of this instability, and finally that existence, regularity and controllability results might be hoped for in the evolution model with a creep term.

The proposed equations might be used numerically as a preprocessing step to restore the 
channel network of digital elevation models. To this aim, a sophisticated digital elevation model (DEM) filtering approach was recently developed in [45], where channels are detected as "curves of minimal effort" where the effort is related to the upstream drainage area and the curvature of isolevel lines. Numerical evidence here will show that simulating landscape evolution carves and potentially restores the channels obstructed by digitization blur and noise. Being able to simulate DEMs may also influence their PDE based encoding [62].

Section 2 is a general review of the constitutive laws of landscape evolution. Formulas from the vast literature are given in Section 2.1 with the notation unified. Then a discussion of the main laws used in the literature is proposed, namely the water conservation and transport laws (Section 2.2), the empirical laws for sediment transport rate (Section 2.3), the sediment mass conservation law (Section 2.4), the "creep," or soil diffusion law (Section 2.5) and, last but not least, the stream incision law (Section 2.6). Section 2.7 then discusses statical models for river configurations, relating stream incision to the problem of finding the optimal channel network needed to minimize a certain energy.

However, this review would not be complete without a thorough analysis of several seminal models proposed in the literature. These models illustrate how the above listed laws combine to reach a complete study of certain real landscapes. Four such models are fully described and analyzed with our unifying notation in Section 3. This section paves the way to the synthesis proposed in Section 4, where it is shown that a system of three partial differential equations linking three state variables (water elevation, sediment concentration and landscape elevation) gives a fairly complete account of all a priori laws proposed in the literature. Section 5 describes the results of several numerical simulations obtained with these constitutive laws. They confirm that all anticipated phenomena (valley formation, drainage network formation) can be simulated on virgin artificial landscapes. From the mathematical viewpoint, the instability under perturbations and nonuniqueness of the landscape evolution is illustrated, while on the other hand the numerical stability of real landscape topographies under realistic values for their evolution is also put to the test.

\section{Constitutive laws}

\subsection{Main notation and formulas}

We start with the customary list of variables. The notation in landscape evolution models is not quite fixed and is sometimes ambiguous. Indeed, some of the variables actually denote macroscopic quantities measured only on one-dimensional channels. Such macroscopic variables are usually denoted by uppercase letters, while lowercase letters will be reserved for functions defined on a planar domain, depending on two geographic coordinates $(x, y)$. For example $Q$ or $Q_{w}$ usually denotes the water flux in a channel (say, a river). The newly introduced variable $\theta(x, y)$ will denote the water height, defined everywhere in the landscape.

Notation (consistent with the majority of references whenever possible). Unless specified as constants, all state variables below depend on time $t$ and on locally planar $(x, y)$ geographic coordinates.

Physical state variables which will be formalized in this manuscript

$z$ land surface elevation (without water)

$S=|\nabla z|$ land surface slope (without water) 
$\theta$ water height, relatable to the channel "hydraulic radius" $\sqrt{Q}$

$z+\theta$ landscape altitude (land surface elevation plus water)

$|\nabla(z+\theta)|$ landscape slope (with water), sometimes also denoted by $S$

$\rho$ sediment density in water

$v$ velocity of flow in channel

Moreover, we write as usual $\nabla \phi$ for the gradient of a scalar variable $\phi(x, y), \Delta \phi$ for its Laplacian, and $\nabla \cdot v$ for the divergence of a vector field $v(x, y)$.

Physical constants, actually the six main model parameters

$u$ ground layer tectonic uplift constant

$c$ creep rate constant

$e$ erosion rate constant

$r$ rain rate constant

$s$ sedimentation rate constant

$m$ erosion exponent

Of course each one of these constants can become spatially variable, depending on a knowledge of the terrain and climate conditions.

Macro variables, somehow empirical

$q, q_{w}$ water discharge per unit width, the law $Q=q^{2}$ is a consequence of an empirical law, according to which the channel width is proportional to $\sqrt{Q}$;

$Q$ total water discharge in a channel (often proportional to $q^{2}$, also interpreted as "transport capacity");

$W, w$ water channel width, usually proportional to $\sqrt{Q}$ or a power of $Q$;

$A$ drainage area, or "contributing area" of a point (or a channel) often equated with $q$, or with $Q$ in the case of a channel;

$q_{s}$ sediment flux caused by water transport;

$\tilde{q}_{s}$ soil flux caused by the hillslope (creep).

The above state variables are linked in the literature by formulas which will be now listed. Most of these formulas will be mentioned in the model discussion, but only those in the second list will be actually involved in our proposition of a physical landscape evolution model. We start with a list of laws that are classified as empirical laws. This means that they have been observed on stationary landscapes, but should some day be deduced from first principles. The second list is a shorter list of (simplified) physical laws governing the motions of water, soil, and sediments, therefore involving the physical variables only.

In the discussion that follows, the land surface and landscape slopes, $|\nabla z|$ and $|\nabla(z+\theta)|$, are both denoted by $S$. The context, however, will distinguish clearly which is being used. The relation and distinction between the two is often an important point, so that using the same symbol likely compensates for this inconvenience.

Main empirical formulas in the literature. All multiplicative constants are omitted. By empirical we mean that the relations are the results of observations on actual landscapes, and therefore are only valid for (some) static or slowly evolving landscapes. They generally link macroscopic variables 
such as the drainage area $A$ of a channel (river) with water discharge $Q$, the channel velocity $v$, its slope $S$ and its length $L$. All of these variables can be (approximately) measured from a DEM and from average flow and altimetry measurements in rivers.

$Q=c A^{m}$ empirical relation between the drainage area $A$ and the water channel discharge, [59], [34], [35], with $m=0.75$ in [52]. This surprising law is only conservative when $m=1$. Indeed, we should expect that a fixed proportion of the collected water flows in the streams (the rest disappearing by evaporation or infiltration);

$v=Q^{m}, w=Q^{b}, \theta=Q^{f}, m+b+f=1, Q=v w \theta$ power laws linking channel width $w$, channel discharge $Q$, and channel velocity $v$ : in [33], where $b=0.26, f=0.4, m=0.34$; $v=c \theta^{2 / 3}|\nabla z|^{1 / 2}$ called Gauckler-Manning formula [16] or sometimes Manning's equation [8]. Here $\theta$ stands for the hydraulic radius, which is proportional to the water height;

$v=C$ according to this empirical law of static landscapes, "Velocity remains essentially constant in the downstream direction at each flood frequency" [35];

$S=A^{-\beta}$ in [14];

$S=Q^{-\gamma}, 0.75 \leqslant \gamma \leqslant 1$ in [24];

$L=C A^{h}, h \approx 0.6$ Hack's Law, an empirical relationship between the length $L$ of streams and the area of their basins;

$Q=S \theta$ (where the velocity of the channel is assumed proportional to its slope).

Main physical laws and models in the literature. All multiplicative constants are omitted. The partial differential equations will be explained in the present section. In the equations that follow, we have standardized the usage of $\frac{\nabla(z+\theta)}{|\nabla(z+\theta)|}$ instead of $\frac{\nabla z}{|\nabla z|}$ for flow direction calculations.

$\Sigma_{i} Q_{i}^{1 / 2} L_{i}$ optimal channel network energy in [52], the sum is on all river branches with lengths $L_{i}$ and flux $Q_{i}$. We will give an overview of this variational model at the end of this section. $\frac{\partial z}{\partial t}=s \nabla \cdot\left(q_{s} \frac{\nabla z}{|\nabla z|}\right)$ Exner's sediment mass conservation equation;

$\frac{\partial z}{\partial t}=s \rho$ Exner equation when only gravity moves the sediment;

$\nabla \cdot(\theta \nabla z)=-r$ water conservation equation in the static case (water flow exactly compensates for rain). Sometimes in this equation $\theta$ is replaced by $q$, and $z$ by $z+\theta$;

$\frac{\partial \theta}{\partial t}-\nabla \cdot(\theta \nabla(z+\theta))=r$ water conservation equation. Water runs off in the direction opposite to the slope at a velocity proportional to the slope. The space and time dependent parameter $r$ is the rain density;

$\frac{\partial z}{\partial t}=k \Delta z+u$ creep diffusion equation, the main equation explaining the observation of rounded hill slopes. The parameter $u$ represents the tectonic uplift;

$\frac{\partial z}{\partial t}=-e \theta^{m}|\nabla(z+\theta)|^{2 m}$ soil erosion equation. The power is often $m \simeq 0.5$. This somewhat empirical equation has two parameters: the soil dependent $e$ erosion coefficient and the model dependent exponent $m$;

$\frac{\partial z}{\partial t}=c \Delta z-e A^{m}|\nabla z|$ in [47].

The last mentioned equation is valid under the assumptions that

(i) all channels are non-alluvial;

(ii) the critical shear stress is zero;

(iii) there is no near-failure contribution to slope erosion (i.e. landslides);

(iv) regolith and bedrock are equally erodible.

In this case the basic evolution equation reduces to a structure where the erosion rate is a linear combination of a diffusive mass-wasting term in Laplacian form, tending to smooth the landscape, 
and a channel erosion term that becomes more important - for a given gradient - as drainage area increases.

The next sections discuss in detail the five main laws recurring in all models, mostly with a (simplified) physical background. All of them are partial differential equations linking the state variables $\theta, z$ and $\rho$.

\subsection{Water conservation law}

The water conservation equation in static conditions with uniform rainfall rate $r$ is $\nabla \cdot\left(\theta \frac{\nabla z}{\nabla z}\right)=-r$ or $\nabla \cdot(\theta \nabla z)=-r$, as it is formulated in Simpson and Schlunegger [59], Smith and Bretherton [60], and Tarboton, Bras and Rodriguez-Iturbe [64], [63]. Indeed, the simplest way to model water transport at the macroscopic level, without reference to fluid dynamic aspects, is by this transport equation in which the water level $\theta$ is transported at a velocity $F$ which only depends on the slope. Then $\frac{\partial \theta}{\partial t}$, the change in water level with respect to time, can be written as a divergence of the water flux, $\theta F$. A source term $r$ (the rain) may also be added. By the first mentioned principle the water runs in the opposite direction of the landscape gradient. The velocity can thus be represented as a function of the slope of the landscape: $F(\nabla(u+\theta))$. The simplest choice is a linear correspondence, so that $F(\nabla(u+\theta))=-\nabla(u+\theta)$. Notice that the landscape altitude $z+\theta$ is defined as the sum of land surface elevation $z$ and of the water height, $\theta$. This gives the first equation in the model,

$$
\frac{\partial \theta}{\partial t}-\nabla \cdot[\theta \nabla(z+\theta)]=r .
$$

where $r=r(x, y, t)$ is the rainfall density at $(x, y)$ and at time $t$. For the sake of simplicity we shall always omit the $(x, y, t)$ dependence in the state equations.

When $z \equiv 0$, one can write the divergence term as $\frac{1}{2} \Delta\left(\theta^{2}\right)$ and the above equation boils down to the porous medium equation. The proposed equation, being in divergence form, is conservative. Water is conserved by the evolution, the only source being $r$, and the only way for water to leave the system is through the boundary of the domain. Clearly, this excludes other phenomena by which rain water can be evacuated or retained, namely evaporation, infiltration, and ice formation or melting.

\subsection{Empirical law of sediment transport rate}

Gilbert [17] reasoned that slope and discharge were the primary factors controlling channel incision and eventually this inference was formalized (e.g., by Smith and Bretherton [60], Kirkby [31] and most subsequent workers) to the sediment transport law

$$
q_{s}=K q^{m} S^{n}
$$

where $q_{s}$ is the sediment transport rate, $q$ is the water discharge, $S=|\nabla z|$ is the surface slope, and $K, m$, and $n$ are constants. Carson and Kirkby [7] and Willgoose, Bras and Rodriguez-Iturbe [70] demonstrate that an equation of the form (2.2) can be developed from alluvial sediment transport theories.

The empirical sediment transport rate equation (2.2) is probably one the most well established laws in geomorphology, being used among many other authors by Howard [25], Loewenherz [38], Tarboton, Bras, Rodriguez-Iturbe [64], Tucker and Bras [65]. Also Moglen, Eltahir, Bras [41] use this law, calling $q_{s}$ the fluvial transport expressed as a power law of the discharge $q$ and the local 
slope, $S$. They mention that the exponent values may range from 0 to 3 for $m$ and from 1 to 3 for $n$. Graf [19] gives the variant $q_{s}=c S+d q^{n} S$. Howard [25] states another variant where $q$ is replaced by the drainage area, but generally $q$ and $A$ are related by a power law, so this variant is equivalent to (2.2). The law reads $q_{s}=K_{q} A^{\alpha} S^{\beta}$, and Howard states that "Processes with $\alpha<1$ will be diffusional, and those with $\alpha>1$ will be concentrative. Creep is modeled with $\alpha=0$ and $\beta=1$. Transport of sand in alluvial channels is concentrative with $\alpha=1.5$ and $\beta=2$." Clearly (2.2) is an empirical law that cannot be used in an a priori model.

\subsection{Sedimentation mass conservation (Exner's law)}

The 3D Exner conservation of mass equation describes both the downslope transportation and the settling of sediment at the interface between the water and land surface:

$$
\frac{\partial z}{\partial t}=s \nabla \cdot q_{s}
$$

with $s=\frac{1}{\epsilon_{0}}, \epsilon_{0}$ being the (constant) packing density of sediment particles and $q_{s}$ the sediment flux. (See e.g. Izumi and Parker [29].) Here it is understood that $q_{s}$ is a vector with three components in $x, y, z$. Indeed $q_{s}$ has a vertical component due to gravity. When this component dominates (i.e. in still water) the above equation can be further simplified as

$$
\frac{\partial z}{\partial t}=s \rho,
$$

with $\rho$ representing the constant concentration of sediment in the water and $s$ a constant. The absence of any $\theta$ dependence is notable in this equation. Physically, this makes sense in that only the sediment in the water closest to the interface matters when considering how much sediment will immediately settle on the land surface.

Exner's law (2.3) is extensively used and often combined with the empirical sediment transport rate equation (2.2). Tarboton, Bras and Rodriguez-Iturbe [64] state that

The basic equations which we regard as sufficient to model the essence of landscape evolution are:

$$
\frac{\partial z}{\partial t}=\nabla \cdot\left[\frac{\nabla z}{|\nabla z|} F(|\nabla z|, A)\right] \quad \text { and } \quad \nabla \cdot\left(\frac{\nabla z}{|\nabla z|} A\right)=1
$$

where $A$ is the upslope area and the second equation is the "calculation of upslope area." The second equation is simply the water conservation and downslope transport equation. The first equation is a combination of Exner's law and a general form of the empirical sediment transport rate equation (2.2), $q_{s}=\frac{\nabla z}{|\nabla z|} F(|\nabla z|, A)$, where $F(|\nabla z|, A)$ ends up being a power law function of slope $(S=$ $|\nabla z|)$ and water flux $(A \simeq q)$.

A deduction that the landscape evolution equation should be put in divergence form $d z / d t=$ $\nabla \cdot\left(Q_{s}\right)+\ldots$ is made in the works of the just mentioned authors, and also Smith and Bretherton [60], Welsh, Birnir and Bertozzi [69], Willgoose, Bras and Rodriguez-Iturbe [70], Dietrich et al. [11], Roering, Kirchner and Dietrich [55], Tucker and Bras [65]. For example Loewenherz [38] writes $\frac{\partial z}{\partial t}=u-\nabla \cdot Q_{s}$, where $Q_{s}$ is the sediment flux and Simpson and Schlunegger [59] give $\frac{\partial z}{\partial t}=\nabla \cdot\left(\left(c+d Q^{n}\right) \nabla z\right)$. 


\subsection{The "creep" law (soil diffusion law)}

In [11], Dietrich et al. commented that

The occurrence of rounded, convex hilltops in badlands puzzled Gilbert and Dutton [18] until Davis [10] commented that repeated dilation and contraction of loose debris on an inclined surface will induce a creeping, downslope transport, and that the effect of this movement in shaping hillslopes is likely to dominate on divides, where surface wash is not concentrated. Gilbert [17] subsequently pointed out that hilltop convexity is common (not just in badlands) and reasoned that creep resulted from disturbance by expansion and contraction, due to freeze-thaw, wet-dry and hot-cold cycles, and biologic activity. He proposed that these processes varied with slope and that a hilltop undergoing steady state erosion should consequently have a convex form. Culling [9] formalized this hypothesis by solving one-dimensional mass conservation equations using a transport law that assumed flux proportional to local hillslope gradient.

\section{Roering [54]}

determined which transport model best preserved morphologic patterns that describe the current landscape form. Models for which sediment flux varies proportionally with hillslope gradient generated broadly convex hilltops inconsistent with the sharp-crested, steep-sided slopes of our study site, whereas the two nonlinear slope-dependent models produced convex-planar slopes consistent with current hillslope form.

These comments refer to a choice for the soil flux $\tilde{q}_{s}$ caused by the hillslope as a function of $\nabla z$ in nonlinear variants of the Culling model $\tilde{q}_{s}=-k \nabla z$.

By Exner's law, the mass conservation equation widely applied in landscape modeling is [11]

$$
\frac{\partial z}{\partial t}=u-\nabla \cdot \tilde{q}_{s}
$$

where $u$ is the uplift. If $q_{s}$ obeys the Culling model, we get the creep diffusion equation which therefore is the main equation justifying rounded hill slopes:

$$
\frac{\partial z}{\partial t}=k \Delta z+u .
$$

Yet several authors [54] since have supported a more realistic model for hill slope soil flux, with a a critical slope $S_{c}$ over which the soil flux blows up and turns into a landslide:

$$
\tilde{q}_{s}=-\frac{k \nabla z}{1-\left(|\nabla z| / S_{c}\right)^{2}},
$$

where $S_{c}$ is the critical slope.

The creep term has been used in landscape modeling extensively to model the deformation of soil under various stresses. The effect tends to be diffusive. While this has typically been represented by a Laplacian smoothing term, the actual effect can perhaps better be described mathematically by the area minimizing equation. The landscape area is the integral $\int \sqrt{1+|\nabla z|^{2}}$ and its gradient descent equation is

$$
\frac{\partial z}{\partial t}=c \nabla \cdot\left(\frac{\nabla z}{\sqrt{1+|\nabla z|^{2}}}\right) .
$$


Making the assumption that $|\nabla z| \ll 1$, the equation becomes

$$
\frac{\partial z}{\partial t}=c \Delta z
$$

which is the usual creep term used in most landscape evolution models. The constant $c$ reflects a diffusion speed depending on soil condition and on the mentioned various perturbing factors (rain splash, wind, chemical weathering, ... ). Such a creep term is found, among others, in Fernandes and Dietrich [13], Moglen, Eltahir and Bras [41], Simpson and Schlunegger [59], Culling [9]. We shall retain (2.6) as one of the fundamental physical equations of landscape evolution. Nevertheless, more sophisticated conservative creep models with a mathematical analysis can be found, for example in the mathematical description of the coupling of weather-limited erosion and a diffusion model given in [67].

\subsection{Stream Incision Law}

The commonly used stream incision law [43] gives the erosion rate of a channel in terms of the flux of water $Q=\theta S$ in the channel and the local gradient. (The relation $Q=\theta S$ links channel flux, slope and height. It assumes a channel velocity proportional to its slope.) The law can be expressed as

$$
\frac{\partial z}{\partial t}=-e Q^{m} S^{n}
$$

where $e$ is a constant, $Q=\theta S$ the water flux, $S$ is the slope, and $m$ and $n$ are exponents. Since $S=\nabla(z+\theta)$, we obtain the erosion law

$$
\frac{\partial z}{\partial t}=-e \theta^{m}|\nabla(z+\theta)|^{m+n} .
$$

Howard and Kerby [27] reasoned that when a channel surface is bedrock, rather than covered by alluvium, the channel's transport ability exceeds supply. They showed that channel incision is limited primarily by the resistance of the underlying bedrock to the boundary shear stress imposed by stream flows. These assumptions led to an erosion model, as compared to a sediment transport law, of a form essentially the same as (2.2). Because the dominant channel forming discharge is a function of drainage area, they argued that discharge, $Q$, could be replaced with contributing drainage area, $A$, which led to an erosion theory that can be written in the form

$$
-\frac{\partial z}{\partial t}=K A^{m} S^{n}
$$

Again, taking a flux law $A=\theta S$, valid in the static case, gives back (2.9). Howard and Kerby analyzed the evolution of a small disturbed area in Virginia and used estimated rates of channel incision to assess the value of $m$ and $n$ in (2.10). According to them the form of the erosion in the channel has the form $d z / d t=-C\left(A^{g} S^{h}-\tau\right)$. Howard [25] mentions the exponents $g=0.3$ and $h=0.7$. Notice that $h \simeq 2 g$. Seidl and Dietrich [26], Dietrich et al. [11], Izumi and Parker [29], Perron, Kirchner and Dietrich [47], and Perron, Dietrich and Kirchner [46] derive a similar erosion model (which also has the creep and the uplift terms)

$$
\frac{\partial z}{\partial t}=c \Delta z-e A^{m}|\nabla z|+u .
$$


This model is referred by Perron et al. to Kirkby [32], Tucker and Bras [65] and Howard [25]. Dietrich et al. [11] have all three terms in their equation: uplift, sedimentation transport term in divergence form, and incision term of the kind proposed by Howard and Kerby [27]. This analysis of all variants leads to the conclusion that a general form of the stream incision law to model the erosion rate, coping with all models, is (2.9). In agreement with [58], we shall adopt in the following $m=n$. Nevertheless, the exponents in this equation are subject to empirical variations not based on physical laws.

\subsection{Statical landscapes via optimal channel networks and branched transport}

Before entering into the details of several evolution models in next section, we summarize here the variational model of the so-called Optimal Channel Networks which has been used in part of the geophysical literature to describe the statical configurations of river basins landscapes. One of the reasons to address these statical models is the recent mathematical analysis that has been done of their "continuous" version, since the goal of the present paper is to suggest rigorous - but rich mathematical models.

A quite comprehensive reference for this topic from the geophysical point of view is [52], even if the specific subject that we want to deal with is mainly developed in [3] (a short previous summary of the same ideas can be found in [2]). Roughly speaking, in these references the configuration of a river basin is described through two main objects: the landscape elevation function $z$, and a river network $N$, that we suppose for simplicity composed by streams bringing water to a single output point $x_{0}$ (say, a lake). A first link between both objects is the fact that at any point the direction followed by water is the direction of steepest descent of $z$. Hence, once we know $z$, we are able to deduce $N$ and, using also the precipitation level $r$, to compute the flux $Q(x)$ at any point $x$, i.e. the quantity of water passing through $x$ while following the steepest descent lines of $z$. As an evolution model ruled by erosion the above references propose the equation

$$
\frac{\partial z}{\partial t}=-Q|\nabla z|^{2}+u
$$

where $u$ is the tectonic uplift. This is a simplified version of the stream incision equation (see (2.9)), where the role of $\theta$ is replaced by that of $Q$ since no physical height of water is considered to affect the shape of the landscape. If instead one considers landscape equilibria, i.e. configurations with $\frac{\partial z}{\partial t}=0$, we get $|\nabla z|=u^{1 / 2} Q^{-1 / 2}$ and this last condition is called the slope-discharge relation. It is explicitly suggested in [3] that in (2.11) one could change the exponents of $Q$ and $|\nabla z|$, thus obtaining different slope-discharge relationships. In general we get $|\nabla z|=c Q^{\alpha-1}$ and the physically interesting case is when the exponent $\alpha$ is very close to $1 / 2$.

To find landscape equilibria a discretization is performed in [3] and a regular square grid is used. Functions defined on the pixels of the grid and vanishing at $x_{0}$ are considered, as well as networks composed by edges of the grid, directed from every point to one of the neighbors. The model requires to find a pair $(z, N)$ satisfying both the slope-discharge condition and the fact that the direction of $N$ is that of steepest descent of $z$. This is not trivial and is a sort of fixed point problem: starting from $z$ one can reconstruct the network $N$ and the flux $Q$ and then define a new function $\tilde{z}$ by integrating the slope $c Q^{\alpha-1}$ along the branches of the network, and then look for $z$ such that $\tilde{z}=z$. Otherwise, one can start from $N$, compute $Q$, define $z$ in the same way and then a new network $\tilde{N}$ by following the steepest descent lines: then the requirement is $\tilde{N}=N$. 
The important idea presented in [3] is the relation between landscape equilibria and Optimal Channel Networks (OCNs in literature, see for instance [53], [49] and [28]). An OCN is a network $N$ minimizing a certain dissipated energy. The dissipated energy in a system satisfying the slopedischarge relation is the total potential energy that water loses in the network. For each pixel we have a quantity of water $Q_{i}$ which falls down towards the next pixel following a length $L_{i}$, and its elevation decreases by a quantity which is proportional to $|\nabla z| L_{i}$ and hence to $Q^{\alpha-1} L_{i}$. Hence, the total energy loss is given by $\sum_{i} Q_{i} Q_{i}^{\alpha-1} L_{i}=\sum_{i} Q\left(x_{i}\right)^{\alpha} L_{i}$. What is proven in [3] is that, if $N$ is an OCN minimizing this energy, then the landscape function $z=z(N)$ reconstructed from it is actually an equilibrium. This actually means that not only the slope of $z$ in the direction of the network is given by $Q^{\alpha-1}$, which is true by construction, but also that this direction is the direction of maximal slope.

Notice that the problems studied in [3] and in the other papers on the subject have undergone a very strong discretization. Compared to continuous models there is a loss of rotational invariance, a fixed scale effect due to the mesh, and several questions concerning the river basin may lose their meaning (for instance questions about the interfaces between two separated parts of the basin and points where the water takes two different directions, or most regularity issues).

Yet, in recent years the mathematical community of optimal transport and calculus of variations developed a continuous counterpart of the network optimization problems of OCNs, called branched transport (see [4] as a general reference for the subject). In this framework, a possibly infinite network $N$ can join two given distributions of mass (in the present case, the rain density $r$ and a Dirac mass $\delta_{x_{0}}$ concentrated at the output point of the network), and the energy associated to this network is $\int_{N} Q(x)^{\alpha} d x$, the integral being performed according to the onedimensional measure on $N$. The role of a landscape function $z(x)$ in this continuous model (where $z(x)=\int_{N(x)} Q(y)^{\alpha-1} d y$, the integral being one-dimensional on the branch $N(x)$ of the network connecting $x$ to the output) has been underlined in [56]. In this paper this function is first shown to be well-defined (an ambiguity could occur at those points $x$ reached by two different branches of the same network); then, it is proven that it satisfies all the properties which were crucial in the discrete case (steepest descent in the direction of the network, above all), and the regularity of $z$ is also investigated. Under the assumption of a minimal rain level $r_{0}$ and supposing $\alpha>1 / 2$ (in dimension 2) it is proven that $z$ is Hölder continuous with exponent $2 \alpha-1$ and that $z$ is never Lipschitz continuous.

More recently, in [6] a different Hölder continuity result is proved for more singular rain distributions (which could be concentrated on lower-dimensional regions), and in [71] a definition is provided and some interesting PDE properties of a landscape function are proved in the case of multiple outputs.

\section{Analysis of four models}

The preceding section can be summarized by stating that there are five main laws considered in the literature for landscape formation:

1. the water conservation and transport law with a velocity proportional to the slope, (2.1),

$$
\frac{\partial \theta}{\partial t}-\nabla \cdot[\theta \nabla(z+\theta)]=r
$$

2. the empirical law of sediment transport rate (2.2),

$$
q_{s}=K q^{m} S^{n}
$$


3. the sedimentation mass conservation (Exner's law) (2.3),

$$
\frac{\partial z}{\partial t}=s \nabla \cdot q_{s}
$$

4. the "creep" law (soil diffusion law) (2.6),

$$
\frac{\partial z}{\partial t}=k \Delta z+u
$$

5. the erosion equation (stream incision law) (2.9),

$$
\frac{\partial z}{\partial t}=-e \theta^{m}|\nabla(z+\theta)|^{2 m}
$$

These five constitutive laws (water mass conservation, sediment rate empirical law, sediment mass conservation, creep law, erosion law) do not appear, to the best of our knowledge, in an ordinate manner in the literature. They have many variants (we mentioned several), and can be used together, or partially together, depending on the scientific goals. Indeed, most papers in geomorphology address, quite logically, the quasi-stationary state of a landscape in slow evolution. In such cases, basic physical laws can be mixed with a posteriori or empirical laws which are only valid for stationary models. Among the above considered five equations, only the second, namely the empirical law of sediment transport rate (2.2), is actually empirical. Thus models which use it will be only valid on quasi-stationary landscapes, and not as a generic landscape formation models.

It is quite licit to perform a landscape stability analysis with laws that are valid only near the equilibrium. Yet, other factors explain the broad variations of the models in the literature. First, many studies actually address a particular landscape, or a type of landscape with specific parameters. Also, models can be made more sophisticated by assuming not three phases (water, sediment, terrain), but rather four (water, sediment, regolith (soft soil), hard bedrock). In that case laws must be introduced describing the rate conversion of bedrock into soft soil. This explains why the four models we shall analyze in this section are not unifiable. It is therefore important to analyze thoroughly such examples before proposing a sort of common denominator to all models. Our introduced model (section 4) will be prone to numerical analysis, and anyway remain extendable or adaptable to the many variants.

\subsection{The Perron, Kirchner and Dietrich model}

This model consists of a single equation. It is not empirical, and uses only the last two mentioned physical laws, the "creep" law (2.6) and the stream incision law (2.9). The creep-erosion competition model is a classic observation going back at least to Gilbert [17]. The principle is that the tops of hills are dominated by sediment transport mechanisms that smooth the land surface, while hillslopes give way to valleys where water runoff becomes concentrated enough to outpace the smoothing processes and incises into the land surface. The simplest model giving an account of this competition in a single equation is proposed in Perron, Kirchner and Dietrich [47]. These authors considered the creep-erosion model and, using topographic measurements on five sites, evaluated the model parameters, particularly $m$. This critical exponent $m$ varies from 0.37 to 0.42 in several landscapes. These authors conclude from their measurements that "the two-process model, despite 
its simplifications, captures the mechanisms that exert the strongest influence on valley spacing in these landscapes." Their equation, derived in detail in [46], is

$$
\frac{\partial z}{\partial t}=c \Delta z-e \theta^{m}|\nabla z|+u .
$$

Equation (3.6) assumes that soil creep flux is proportional to the local topographic gradient, and that the rate of erosion by channelized flow of water is proportional to the rate of energy expenditure. The model of Perron et al. utilizes the drainage area $A$ rather than $\theta$, but we adopt the static flux law $A=\theta S$, as done earlier.

The derivation of (3.6) uses the Gauckler-Manning formula [16] $v=-c \theta^{2 / 3} \nabla z^{1 / 2}$, where $c$ is a constant related to the terrain roughness, and $\theta$ replaces the "hydraulic radius" of the channel, which can be interpreted roughly as the height of water, being the ratio of the channel cross section area by its wet perimeter. $|\nabla z|$ stands for the terrain slope (denoted by $S$ here and in the literature).

This paper [47] intended to show that the ridge-valley wavelength can be predicted from erosional mechanics and that "the characteristic ridge-valley wavelength is an emergent property of the erosion and sediment transport processes that shape the landscape." Strikingly enough (3.6) appears in other derivations and with other arguments, see e.g. [25], where the exponents are, however, different.

\subsection{The Willgoose, Bras and Rodriguez-Iturbe model}

The Willgoose et al. model [70] is far more complex, with 30 parameters or state variables in the notation list. It gives for example explicit equations for a logical variable triggering the formation of a channel. Therefore the landscape evolution law is different in channels and on hillslopes. Nonetheless, the model is based on the constitutive laws we have discussed, namely the empirical law of sediment transport rate (2.2) coupled with the sedimentation mass conservation (Exner's law) (2.3), and the "creep" law (soil diffusion law) (2.6). On hillslopes, it is the creep equation (2.6) with uplift,

$$
\frac{\partial z}{\partial t}=u+c \Delta z
$$

while in channels the equation reads

$$
\frac{\partial z}{\partial t}=u+c \Delta z-d \nabla \cdot\left(\theta^{m}(|\nabla z|)^{n-1} \nabla z\right)
$$

In the notation of this paper the sediment flux is $q_{s}=q^{m} S^{n}$ where $q$ is the water discharge per unit width, which we can therefore interpret as $\theta . S$ is the slope, but since this paper takes its divergence, we must rather interpret $S$ as $-\nabla z$. Thus, the equation in channels is $\frac{\partial z}{\partial t}=u+e \nabla \cdot\left(q_{s}\right)$, where we omit several terrain dependent constants.

Since the paper is ambiguous on that point, we interpreted that the slope $S$ used in the paper is a vector opposite to $\nabla z$. Yet, no matter how we interpret this equation, it raises serious concerns. If the sign is positive in the last term of (3.7), then it becomes a diffusion term for sediments in the channel, whose diffusion speed increases with the water discharge. To increase the diffusion of the soil in the presence of water cannot explain valley formation. If both diffusion terms are positive in the equation, the steady state solution of the equation will for example be a constant under Neumann boundary conditions. If instead the sign is negative, the equation is ill-posed as 
an inverse heat equation. This seems contradictory to the intent of the model, while removing the divergence in the equation would give back a variant of (3.6). The same instability was noticed by Welsh et al. [69] about the Smith-Bretherton model, which contains the same reverse diffusion equation:

The problem that Smith and Bretherton ran into was that all reasonable hillslopes were unstable in their model. This instability was similar to the backward heat equation, where the modes associated with the highest frequencies grow the fastest and this precluded any numerical analysis of the nonlinear SB model.

\subsection{The hybrid Smith and Bretherton model}

We can follow Welsh's [69] comments on the Smith Bretherton model [60], which appears to be a typical hybrid model where the empirical a posteriori observed laws are mixed with physical conservation a priori laws. Here we have two state variables, the elevation $z$ and the water depth $\theta$. The a priori laws are that the water height $\theta$ obeys the water conservation law (2.1), and runs downslope:

$$
\frac{\partial \theta}{\partial t}=r+\nabla \cdot\left(\frac{\nabla(z+\theta)}{|\nabla(z+\theta)|} q_{w}\right),
$$

and Exner's law of sediment conservation (2.3), together with the assumption that sediment is transported by the water downslope:

$$
\frac{\partial z}{\partial t}=u+\nabla \cdot\left(\frac{\nabla(z+\theta)}{|\nabla(z+\theta)|} q_{s}\right) .
$$

Both equations translate the physical laws and the classic assumption that water and sediment run downslope. Yet, the above equations do not specify what the water and sediment fluxes $q_{w}$ and $q_{s}$ are: these are specified by empirical a posteriori laws. These laws are based on observations of quasi-static landscapes and are therefore only valid near equilibrium. Then, replacing $q_{w}$ and $q_{s}$ with the empirical sediment flux the above equations become

$$
\begin{aligned}
\frac{\partial \theta}{\partial t} & =r+\nabla \cdot\left[\theta^{5 / 3} \frac{\nabla(z+\theta)}{|\nabla(z+\theta)|^{1 / 2}}\right], \\
\frac{\partial(z+\theta)}{\partial t} & =u+\nabla \cdot\left[\theta^{10 / 3}|\nabla(z+\theta)|^{2} \nabla(z+\theta)\right] .
\end{aligned}
$$

Provided the empirical a posteriori laws that have been plugged into the physical equations are correct, these equations can be used (e.g.) for stability analysis.

\subsection{The Tucker and Slingerland model}

We shall end this study of four complete models with the more complex of all. It (realistically) introduces three landscape layers instead of two, namely bedrock, regolith (a layer of loose, heterogeneous material covering solid rock, which includes dust, soil, broken rock, etc.), and water.

In Tucker and Slingerland's [66] "physically based model of drainage basin evolution," called the GOLEM model, basin evolution is simulated under the action of weathering processes, hillslope transport, fluvial bedrock erosion and sediment transport. The model has the three above-mentioned 
layers: bedrock, regolith and water. We give the equations with the usual conversion to our notation. Here the elevation $z=b+r_{e}$ is the sum of the bedrock height and of the regolith thickness. The model has a conversion rate from bedrock to regolith by weathering. At some points, the bedrock can be denuded, and there erosion occurs. On the regolith, instead, there is a sedimentation-transport term which can deposit or remove regolith, but the flux only depends on the water height and on the slope. The equations for bedrock $(b)$ and regolith $\left(r_{e}\right)$ evolution are

$$
\begin{gathered}
\frac{\partial b}{\partial t}=u-\omega-e\left(\theta^{2 / 3}|\nabla z|^{2 / 3}-\tau_{c}\right)^{+} ; \\
\frac{\partial r_{e}}{\partial t}=\omega+\nabla \cdot\left[\left(\theta|\nabla z|-\tau_{c}\right)^{+}\right]+c \Delta\left(b+r_{e}\right),
\end{gathered}
$$

where $\tau_{c}$ is a threshold above which bedrock channel erosion is triggered, $\omega=e^{-r_{e} / r_{e, 0}}$ is a conversion rate from bedrock to regolith which decreases quickly if the regolith is thick.

There were several issues in transcribing these equations from the original paper. First of all the diffusion term due to regolith creep had the wrong sign in the paper, being a reverse diffusion $-\Delta\left(b+r_{e}\right)$, and we have changed this sign in conformity with the literature which defines creep as a diffusion. This confusion is caused by the use in this paper (and in several others) of the notion of "downslope gradient," which is opposite to gradient. For the second term on the right-hand side of (3.9), we also replaced the slope $S$ with $|\nabla z|$, in conformity with the usual mathematical convention that $\nabla z$ is the upslope gradient. We interpreted the state variable $Q$ in the original model as $\theta^{2}$, because the model gives $W=k_{c} \sqrt{Q}$ where $Q$ is the water discharge and $W$ the channel width. Thus the water height $\theta$ is also proportional to $\sqrt{Q}$. We also simplified in the expression of $Q_{s}$ the term $\left(\tau-\tau_{c}\right)\left(\sqrt{\tau}-\sqrt{\tau_{c}}\right)$ into $\left(\tau^{3 / 2}-\tau_{c}^{2 / 3}\right)$. This does not change the meaning of the model and yields a simpler expression for (3.9).

Another notation issue is that in this model $\nabla Q_{s}$ likely means $\nabla \cdot Q_{s}$ in order for the dimensions to be consistent. But the direction of $Q_{s}$ being unspecified (it is somewhat treated as a scalar), we assign its direction to be $\frac{\nabla z}{|\nabla z|}$ as usual. In the same way, $Q$ is treated as a scalar in the model, since the formulas take its square root. We thus also assign its direction to be $\frac{\nabla z}{|\nabla z|}$. Running through the above corrections, we obtain

$$
\frac{\partial r_{e}}{\partial t}=\omega+k \nabla \cdot\left[\left(\theta|\nabla z|-\tau_{c}\right)\right]+c \Delta\left(b+r_{e}\right) .
$$

Now the equation appears mathematically consistent. But the general meaning of this model is that the sediment flux $Q_{s}$ will be active only when a certain threshold $\tau_{c}$ is reached. Mathematically, it results in the equation

$$
\frac{\partial r_{e}}{\partial t}=\omega+k \nabla \cdot\left[\left(\theta|\nabla z|-\tau_{c}\right)^{+}\right]+c \Delta\left(b+r_{e}\right) .
$$

This paper gives the model equations in one piece. However, nothing guarantees in the model several consistency properties (which must therefore have been implicitly enforced in the computer simulations):

- the regolith layer $r_{e}$ should stay nonnegative, but nothing in the second equation prevents it from becoming negative; 
- the sediment flux is nonconservative. In other terms, we have a transport term $Q_{s}$ for sediments but it does not correspond to actual sediments pried away by erosion;

- there is no erosion term for regolith, only transport and diffusion terms. It is, however, clear that the soft soil can undergo an erosion of the kind only given to bedrock.

Furthermore, the sediments are transported by water, and the water flux is named $Q$ in the model (which translates in $\theta^{2}$ by our explanation above). Thus the model is incomplete without specifying that $\theta$ is as usual proportional to $A$, the drainage basin area, and $|\nabla z|$.

\section{Derivation of three main constitutive equations}

Of the four models described in the previous section, the Willgoose, Bras, and Rodriguez-Iturbe model (Section 3.2) and Tucker and Slingerland model (Section 3.4) are not designed for the performance of mathematical analysis or numerical simulation, but only for the derivation of secondary laws and for observations on specific landscapes. On the other hand, the Perron et al. model (Section 3.1) and Smith and Bretherton model (Section 3.3) are definitely too simple to cope with the variety of landscapes, as Perron et al. acknowledges: it is designed to model the regular valley spacing observations in some landscapes. Nevertheless, all models point to a common denominator with an intermediate complexity. This model should manage the three main observable physical variables, namely the actual landscape elevation $z(x, y, t)$, the water elevation $\theta$, and the sediment concentration in water $\rho$. To model the interaction of these variables, we need to know how water runs on the landscape, how the landscape is eroded by water, how the eroded sediment is transported and deposited; and finally how the landscape weathers under the combined effects of landslides, chemical erosion, rain splash, etc. These effects lead to a closed minimal model, where other interactions and complications will be neglected. The forgotten effects are:

o water evaporation;

- infiltration (which would require a volumetric ground model. From that viewpoint we are very far from volumetric modeling of stratified landscapes as the one proposed in [39]);

- conversion of bedrock in regolith (which would require a fourth state variable, the elevation of the regolith);

- ice formation (glaciers) which would require the addition of a fifth state variable.

In this section, the above mentioned landscape evolution principles will be translated into single equations. Then the equations will be assembled for the final model treating the main independent variables, namely the water level $\theta$, the land surface elevation $z$, and the sediment concentration $\rho$. We call landscape altitude the sum $z+\theta$.

The basic mechanism is that as water flows, it pries particles of sediment loose from the terrain, which mix with the water. According to [60], "drainage surfaces are self-forming through the basic mechanisms of sediment transport and erosion." At the same time, some particles of sediment from the water settle back onto the terrain layer due to gravitational forces. Finally, the gravitational forces acting on the plastic soil, and several corrosion factors such as rain splash, wind, contractions and dilation caused by physical factors and chemical corrosion lead to an overall smoothing out of the landscape, which is usually called "creep." According to G. K. Gilbert [17], "In creep the chief disturbing agencies are expansion and contraction, and these are caused by freezing and thawing, heating and cooling, wetting and drying." He also mentions the agitation due to earthquakes. In short, the simplest complete model for landscape evolution must contain terms representing the following effects: rain, water flow by gravity, water erosion, sedimentation and creep. While keeping in the model all of these effects, simplicity will be sought by attempting to describe the main 
dynamics of landscape evolution with the fewest parameters and the simplest equations. Gathering the various agents and principles of their interaction as stated in the literature, leads to the following list of landscape evolution principles, which will be further formalized:

1. Water runs with a direction and speed opposite and proportional to the landscape gradient [60]: "Water flowing over the surface moves down the gradient of the surface." (In other terms, inertial effects of water velocity and more generally all complex fluid mechanics effects are neglected);

2. Erosion grows with the speed of water and with the height of water;

3. Exner's law: The sedimentation rate is proportional to the concentration of sediment in water;

4. Several area erosion factors (chemical weathering, rain splash, wind, gravity) cause soil "creep", namely a trend of the landscape to minimize its own surface area. This evolution will be expressed by a simple diffusion term.

5. Conservation laws for sediment material and for water apply.

We have seen in Section 2 that the first four principles result in four equations, namely (2.1), (2.9), (2.4), and (2.8). The fifth is described by (4.3) in the next section. Our goal now is to design a synthetic model compatible with the literature, where the a posteriori empirical laws such as the empirical law of sediment transport rate (2.2) have been set aside.

\subsection{A possible simple general model}

The above mentioned equations are combinable, but there is a missing link. Nowhere do the laws state where the transported sediment comes from: obviously it comes from erosion, and this gives an additional equation which seems to have been neglected in the literature. One can phrase this last equation as a conservation law: the change in the amount of sediment in water, $\frac{\partial}{\partial t}(\theta \rho)$, plus the change in the land surface elevation due to erosion and sedimentation (neglecting creep and uplift, which are not water-dependent effects), plus the change in sediment amount due to water transport must be 0 . Thus,

$$
\frac{\partial}{\partial t}(\theta \rho)+\left(\frac{\partial z}{\partial t}-c \Delta z-u\right)-\nabla \cdot(\theta \rho \nabla(z+\theta))=0 .
$$

By combining the creep, and erosion and sedimentation equations (2.8), (2.9) and (2.4) and keeping the water flow equation (2.1) and the sedimentation equation, we obtain a system of three partial differential equations in the state variables $(\theta, z, \rho)$,

$$
\begin{gathered}
\frac{\partial \theta}{\partial t}-\nabla \cdot[\theta \nabla(z+\theta)]=r \\
\frac{\partial z}{\partial t}=c \Delta z-e \theta^{m}|\nabla(z+\theta)|^{2 m}+s \rho+u, \\
\frac{\partial}{\partial t}(\theta \rho)+\left(\frac{\partial z}{\partial t}-c \Delta z-u\right)-\nabla \cdot(\theta \rho \nabla(z+\theta))=0,
\end{gathered}
$$

where $r=$ rain, $c=$ creep speed, $s=$ sedimentation speed, $e=$ erosion speed, $u=$ tectonic uplift.

As we have mentioned the erosion exponent $m$ is usually taken in the range $\left[\frac{1}{3}, 1\right]$. The term "sediment concentration" might suggest that $0 \leqslant \rho \leqslant 1$. This requirement is nevertheless not necessary, and has no physical basis since $\rho$ is not expressed as a percentage. However, there is a carrying capacity for sediment in water, beyond which the water mechanics will be altered. Clearly a mud flow does not have the same physical properties as water carrying light sediments. Thus, $\rho$ cannot become too large, but this "transport limited" feature is not directly enforced in the above 
model. Nevertheless, the second equation states that the sedimentation rate increases with sediment concentration $\rho$.

The sedimentation-erosion-creep equation gives an a priori quasi-static law. Notice that the essential equation of the model is (4.2), because it contains all effects and parameters linked specifically to the type of landscape.

The sediment and creep speeds are in competition for similar effects: smoothing the landscape. The landscape evolves in a different way when each is changed. While creep tends to cause local smoothing, sedimentation effects can act more non-locally. On a large slope, much of the eroded sediment up-slope is carried down-slope, where there is a large moving sedimentation front. This sedimentation front is observed in Nature close to ocean shores. This particular geological phenomenon is not taken into account in the models outlined in Section 3. In these models, a channel has no "memory" of sediments eroded upstream.

\subsection{The actual number of parameters of the minimal model}

The above equations, though being somewhat a minimal model, exhibit nevertheless six parameters! Exploring a six parameter space is impossible, even when guided by intuition. Thus, it is important to derive relations between parameters so that some can be eliminated. Actually the uplift parameter is irrelevant, in the sense that it simply changes the average landscape level. Thus a numerical simulation can definitely omit it. A second observation is that setting (say) $c=1$ amounts to fixing the creep speed. Since the other landscape evolution factors (erosion and sedimentation) can instead vary, fixing $c$ does not affect the exploration of parameters, a small $c$ corresponding to larger $e, s$ and $u$ and conversely. Nevertheless, although a default value for $c$ can be adopted, it is better to keep $c$ as a parameter to allow for a simplified model with no creep at all $(c=0)$. A last observation reducing the parameter exploration is the following.

The relative orders of magnitude of $\theta$ and $z$ are an important feature to control. To avoid "flooding" $r$ must remain small with respect to $z$. On a scale of 0 to 1 for $z$ and $\nabla z$, the realistic orders of magnitude for $\theta$ and $\nabla \theta$ are $10^{-3}$ or less. It follows that (4.1) is almost linear. In other terms, multiplying $r$ by $\lambda$ amounts to multiplying $\theta$ by the same factor. But multiplying $\theta$ by a factor $\lambda$ in (4.2) is, to first order, equivalent to multiplying the erosion factor by $\lambda^{m}$. Thus, one can expect similar effects on the shape of the landscape by varying indifferently $r$ or $e$.

Last but not least numerical experiments will show that only values of $m$ between 0.3 and 0.6 give realistic landscape shapes. Putting these heuristics together leads to restrain the initial six parameters space to a smaller $(r, s, m)$ space, where in addition the range for $m$ is quite limited.

This actual dependence on only a few parameters may explain why so many digital elevation models have a similar aspect.

\section{Numerical experiments}

This section shows preliminary numerical results obtained by the minimalist three-equation model (4.1)-(4.3) on various initial landscapes and for various parameters. To get rid of the complex discussion on boundary conditions, we have performed the experiments on a synthetic "island," so that the boundary conditions are straightforward. For $\theta$ we adopted the Dirichlet boundary condition $\theta=c$ on the boundary (where $c$ is some constant ocean level). For sediment we adopted the classic Neumann boundary condition. The choice for the boundary condition for the landscape is to extend 
the slope across the domain boundary. For example, we set $z(-1, j)-z(0, j)=z(0, j)-z(1, j)$ on the left side of the domain. Experiments on pieces of landscapes that are not whole islands or continents would be of course questionable, as they do not contain the correct information on water inflow on the boundary. This is why in the literature the evolution simulation is often restricted on a given basin. Yet, this choice still is not quite satisfactory: the basin shape also evolves with time. In any case, the experiments here must be understood as first numerical trials on the above proposed three-equation system. Their goal is to show the variety of effects that can be reached with even a simplistic model. We shall show experiments on simple artificial landscapes, and also experiments on real landscapes encoded as digital images (DEMs). From that point of view, the experiments herewith fall naturally into the realm of image processing and particularly as a particularly well founded application of partial differential equations to digital images [1]. Indeed, landscapes are $a$ priori modelled as digital images without local minima (counting that these are filled in by lakes), which make them a particular class of images.

The simulated examples will illustrate some common geomorphological features produced by Nature and observed in the literature of landscape formation. These features include the inception of river networks [5, 29, 69, 70], the convex upslope-concave downslope appearance of hills [10, 17], the regular spacing of river networks [38, 47,60], the sedimentation front at tributary junctions (where flow is considerably slowed) [51], fractal river branching [52], and basin expansion [20]. The numerical study is challenging because of the subtle numerical interaction between a thin water layer concentrated mainly on a river network, and the singularity it actually creates in the landscape. The discussion on the numerical schemes is far from trivial and will be published as a separate work. To make a long story short, the schemes are explicit finite volume schemes for the transport equations (4.1) and (4.3), and an upwind finite difference scheme for the erosion-sedimentation equation (4.2).

A quick exploration of the parameter space will show that several of these parameters strongly influence the landscape morphology, in particular its valley spacing. The model shows promise in the field of channel network restoration, as river networks tend to become clearer with the proper choice of parameters in the erosion model. A first question, once a set of parameters is chosen, is how to fix a stopping time that will be as much as possible independent of a time scale, to be able to compare the effects of each parameter. A rule that seems to give reliable and interpretable results is to stop the landscape evolution when $20 \%$ of the initial landscape volume has been eroded and transported. This amount is quite sufficient to observe significant alterations and their structure. In all initial synthetic experiments, the initial landscape is a two-dimensional Gaussian ranging between 0 and 1 . We shall not discuss a realistic normalization of the parameters. Thus the values for the rain, erosion, creep, and sedimentation constants indicated in the experiments are given in a convenient numerical scale, not in a physical scale. Nevertheless, the elevation $z$ and water height $\theta$ parameters must be related. The initial maximal elevation is $z=1$ and the minimal is 0 . The rain parameter $r$ is instead measured in multiples of $10^{-7}$. Thus $r=100$ means in fact $r=10^{-5}$ in the same scale as $z$.

Figure 1 shows the time evolution of a mountain with noise added. This example illustrates the time evolution of features such as river networks. The sensitivity of such features to the parameters will be exhibited in several examples. This figure shows for once the landscape in perspective view. (In all following figures, the elevation $z$ will be rendered in a grey scale.)

The first obvious observation is the digital elevation model (DEM) sampling issue. DEM's fall short of having the right resolution to give the correct slope and the correct channel network at pixel 

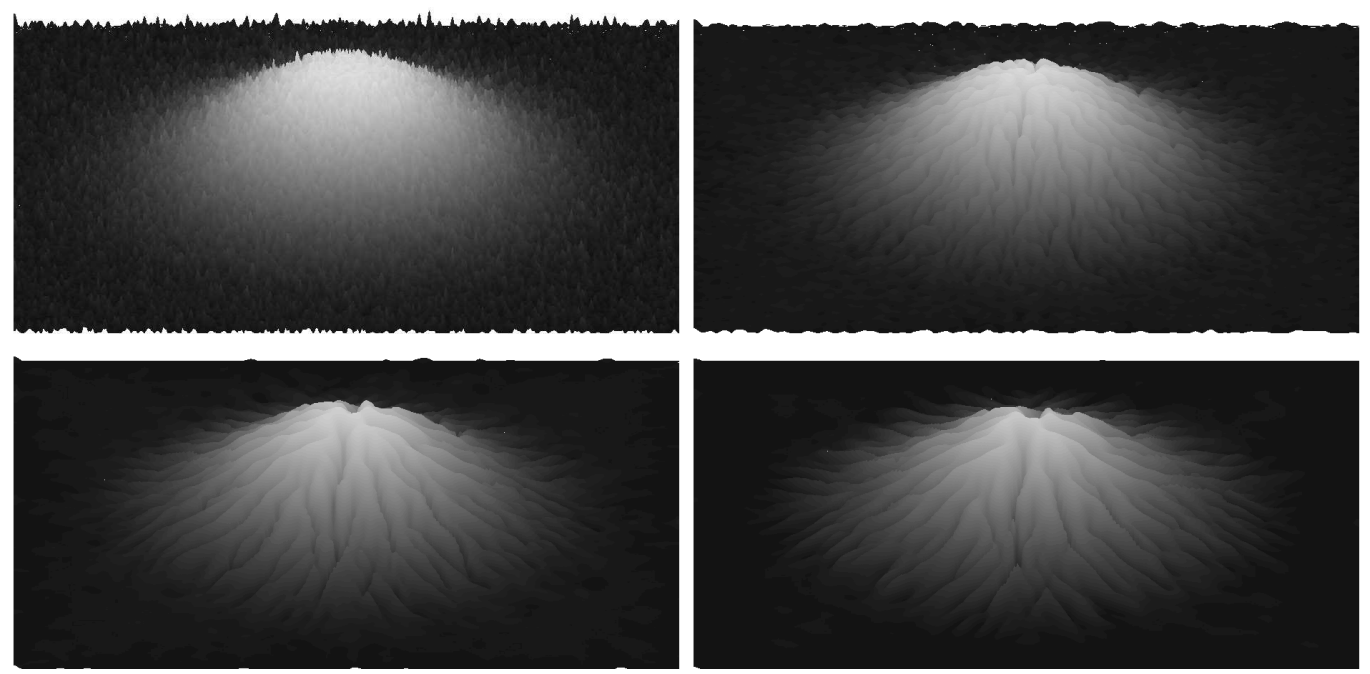

FIG. 1. Landscape evolution in time (perspective view). Top left to Top right: Initial landscape; 1,500 iterations. Bottom left to bottom right: 4,500 iterations; 7,500 iterations.

scale. Research on the exploitation of DEM's shows that the first task is actually to restore their water channel network [30, 44].

These obvious observations are confirmed by experimentation on the influence of resolution of the initial DEM on the river network. For the very same evolution equations, a higher DEM resolution gives a denser river network. Channel branching is not observed for lower resolution landscapes (See Figure 2). This branching becomes more pronounced for higher resolution landscapes, showing a fractal structure. These experiments are performed on a slightly noisy Gaussian DEM defined on a square domain.

Another expected observation is the high sensitivity to initial conditions. In principle for a perfectly radial initial condition like a Gaussian, one can conjecture the existence of a radial solution. Nevertheless, it may also be conjectured that such a solution is unstable, an energy saving water transport being assured when water gathers in channels. As a matter of fact, without any additional noise, channelization occurs due to the quantization of the Gaussian image. With the addition of (small) levels of noise, the resulting channel network becomes less and less symmetric and shows a high variability. Figure 3 shows a comparison of the evolution on various levels of initial noise. The level of channelization is similar, except that the higher noise levels show greater branching and less symmetry in their networks.

Figure 4 shows the result of landscape evolution on a Gaussian with $N\left(0,(10 / 255)^{2}\right)$ noise added. The parameters of rain, erosion, creep, and sedimentation have been halved and doubled to show the influence of each one on the evolution of the landscape. These parameters are discussed further in the following experiments. The main observation here is that each one of the parameters by itself influences strongly the landscape evolution. We conjectured in Section 4.2 that the variation of the rain $r$ and erosion $e$ parameters should have the same influence on the landscape morphology. This fact is confirmed here. In the second row, the first and the third landscape results are fairly identical; the first has been obtained by doubling $r$ and the second by doubling the erosion parameter 

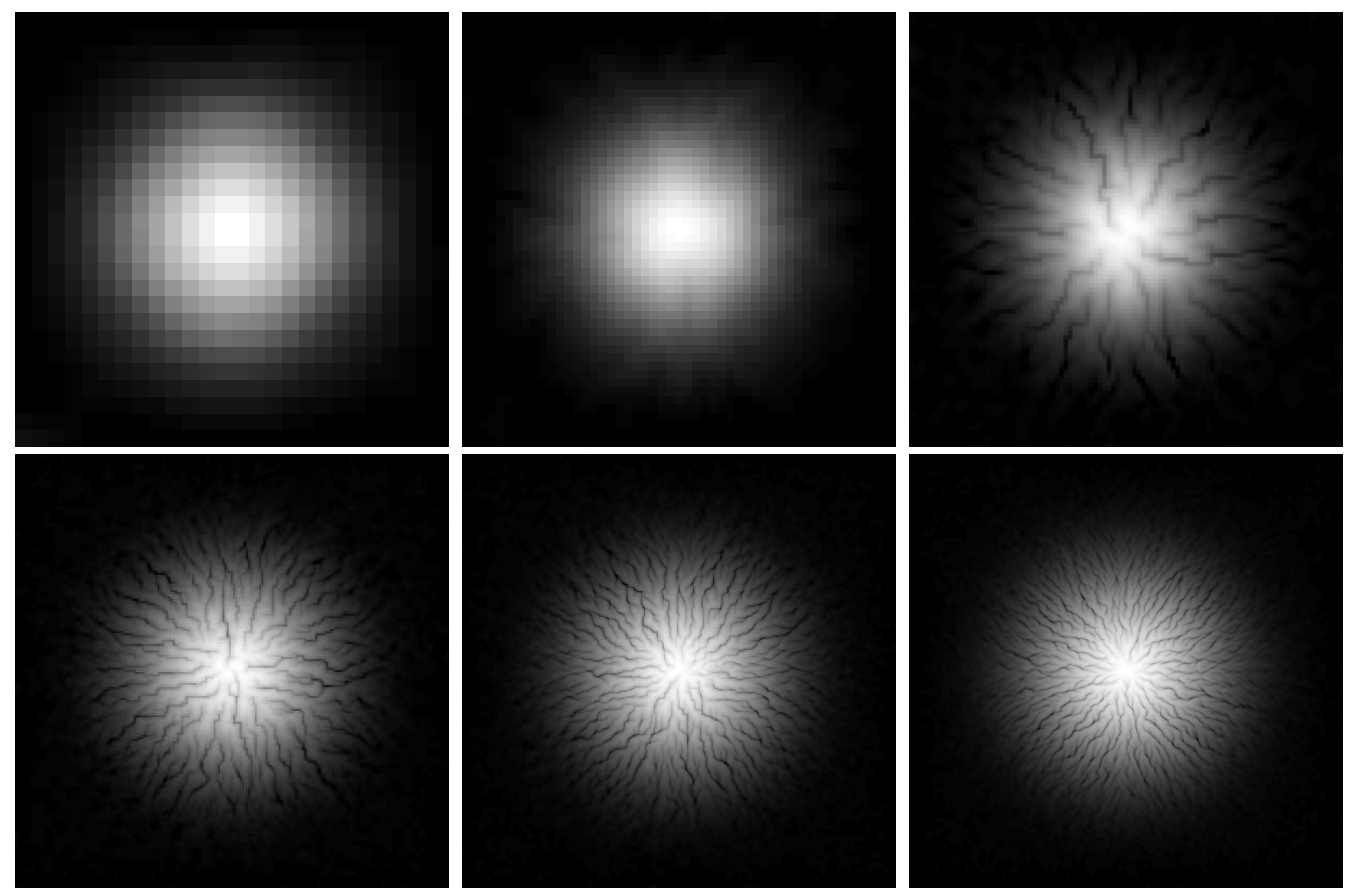

FIG. 2. Landscape evolution on various resolutions of the same landscape. (Here and in all following figures, the elevation $z$ is rendered in grey scale.) Each initial landscape is a (sub-sampled) Gaussian with $N\left(0,(10 / 255)^{2}\right)$ noise added. Parameters: $r=100, e=3, c=1, s=1$. Top left: $26 \times 26$; Top right: $51 \times 51 ; 101 \times 101$; Bottom left to bottom right: $201 \times 201 ; 301 \times 301 ; 401 \times 401$.
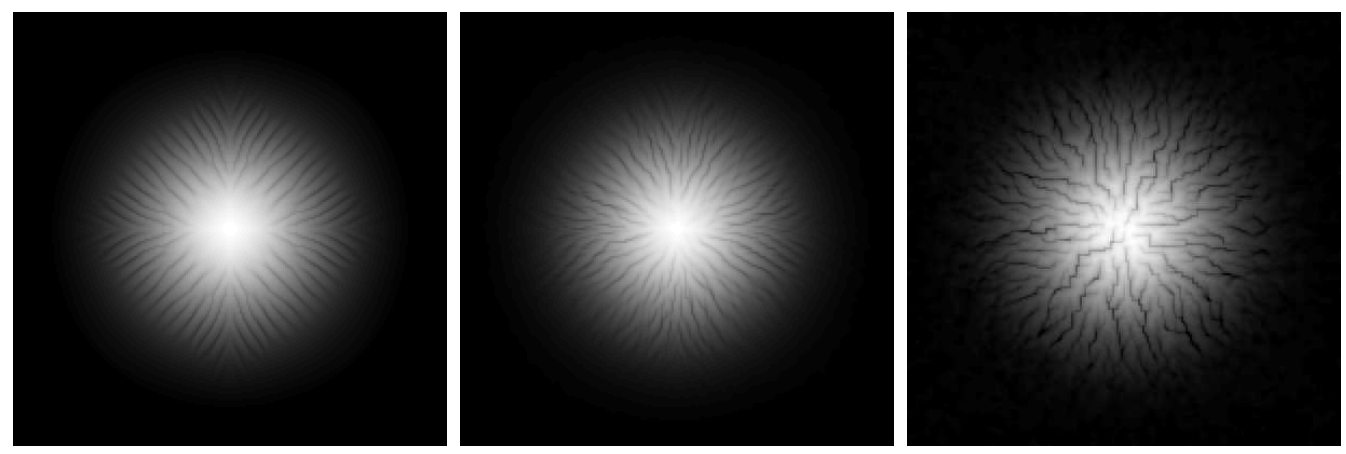

FIG. 3. Landscape evolution on an initial $201 \times 201$ landscape, a Gaussian with various amounts of noise added. Parameters: $r=100, e=3, c=1, s=1$. Left: No added noise. Middle: $N\left(0,1 / 255^{2}\right)$ noise added. Right: $N\left(0,(10 / 255)^{2}\right)$ noise added. This experiment illustrates the strong sensitivity to initial conditions.

$e$. For the same reason, the third result in the first row and the second in the second row are also almost identical. They have been obtained by halving $r$ and $e$ respectively. The third row shows the influence of the creep parameter $c$. As expected, and shown in [47], the creep parameter is 

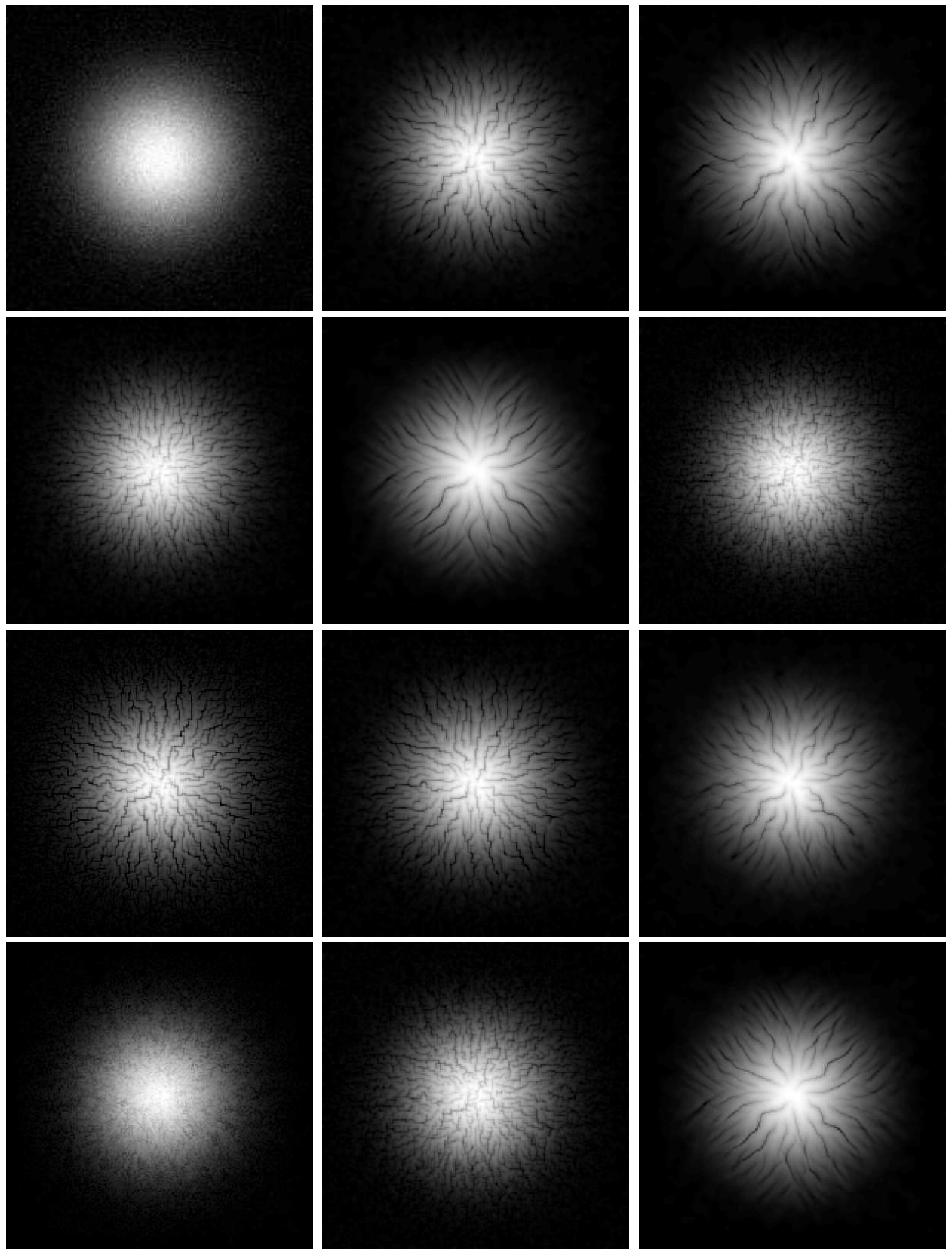

FIG. 4. Influence on the landscape evolution of each parameter. The initial $201 \times 201$ landscape is a Gaussian with $N\left(0,(10 / 255)^{2}\right)$ noise added. Top left to top right: Original image; the base case of $r=100, e=3, c=1, s=1$; the same but with $r=50$. Second row: Double rain; half erosion; double erosion; 3rd row: 0 creep; half creep; double creep; Last row: 0 sedimentation; half sedimentation; double sedimentation. 

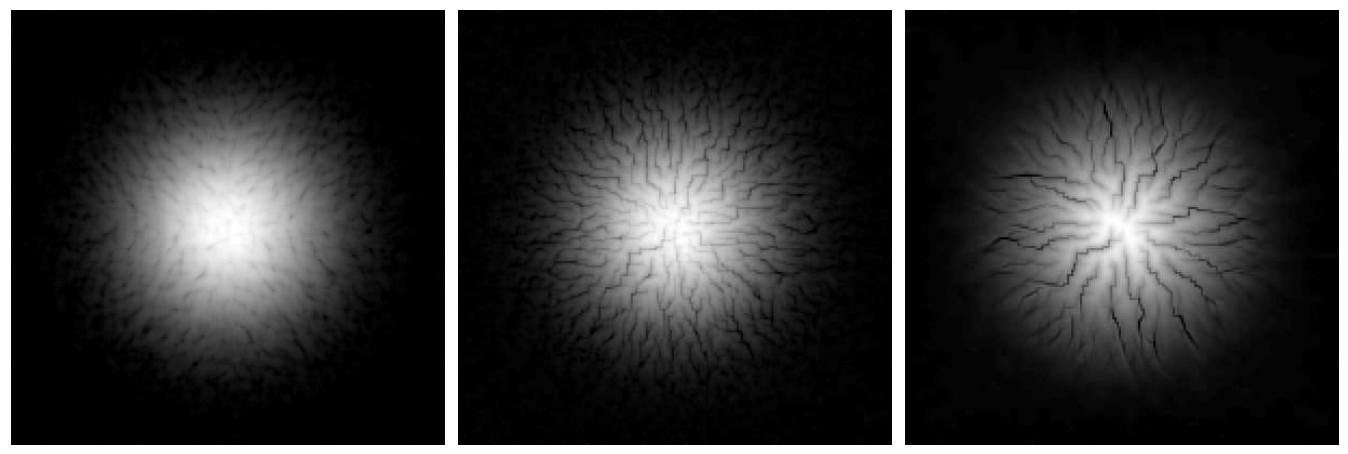

FIG. 5. Landscape evolution on an initial $201 \times 201$ landscape, a Gaussian with $N\left(0,(10 / 255)^{2}\right)$ noise added. Parameters: $r=100, c=1, s=1$, and variable erosion $e$ and erosion exponent $m$. The erosion coefficient has been adjusted according to the erosion exponent for parameter balance issues. A higher erosion exponent tends to give straighter rivers. Left: $e=0.01, m=0.1$; middle: $e=1, m=0.4$; right: $e=100, m=0.8$.

supposed to smooth out the landscape and therefore to increase valley spacing. This supposition is experimentally confirmed. Probably the most surprising result is obtained in the last row, where it is apparent that the sedimentation rate $s$ also influences valley spacing. In short, increasing either rain $r$ or erosion $e$ increases valley spacing, while increasing creep $c$ or sedimentation $s$ decreases it.

The influence of the exponent $m$ in the erosion equation (4.2) is the last to be explored. (We have seen authors proposing values $m$ ranging from 0.3 to 1.) It can be expected that a larger $m$ will favor erosion in deep channels, while a smaller $m$ is more temperate. Thus, as confirmed by the experiments in Figure 5, one expects and observes an almost uniform erosion if $m$ is very small, and more and more incised channels when $m$ grows. Increasing the erosion exponent gives a less dense channel network. Notice that the erosion coefficient had to be changed simultaneously in order to maintain an approximately equal number of iterations.

Table 1 summarizes the results shown in Figures 4 and 5, and shows the effect of increasing each parameter (keeping the others fixed) on channelization levels.

This preliminary study suggests that varying the climatic conditions (the rain) can change the morphology of the landscape. One should therefore expect to observe landscapes with hybrid shape, having traces of two different rain regimes in their channelization. The experiment shown in Figure 6 illustrates this possible phenomenon. Continuing a simulation with the same parameters on a mature initial landscape usually results in a morphology similar to the initial condition: compare results 2

TABLE 1. A summary of the effect increasing each parameter has on channelization

\begin{tabular}{ll}
\hline Parameter & Effect of Increase \\
\hline rain $r$ & more channelization \\
erosion $e$ & more channelization \\
creep $c$ & less channelization \\
sedimentation $s$ & less channelization \\
erosion exponent $m$ & less channelization \\
\hline
\end{tabular}



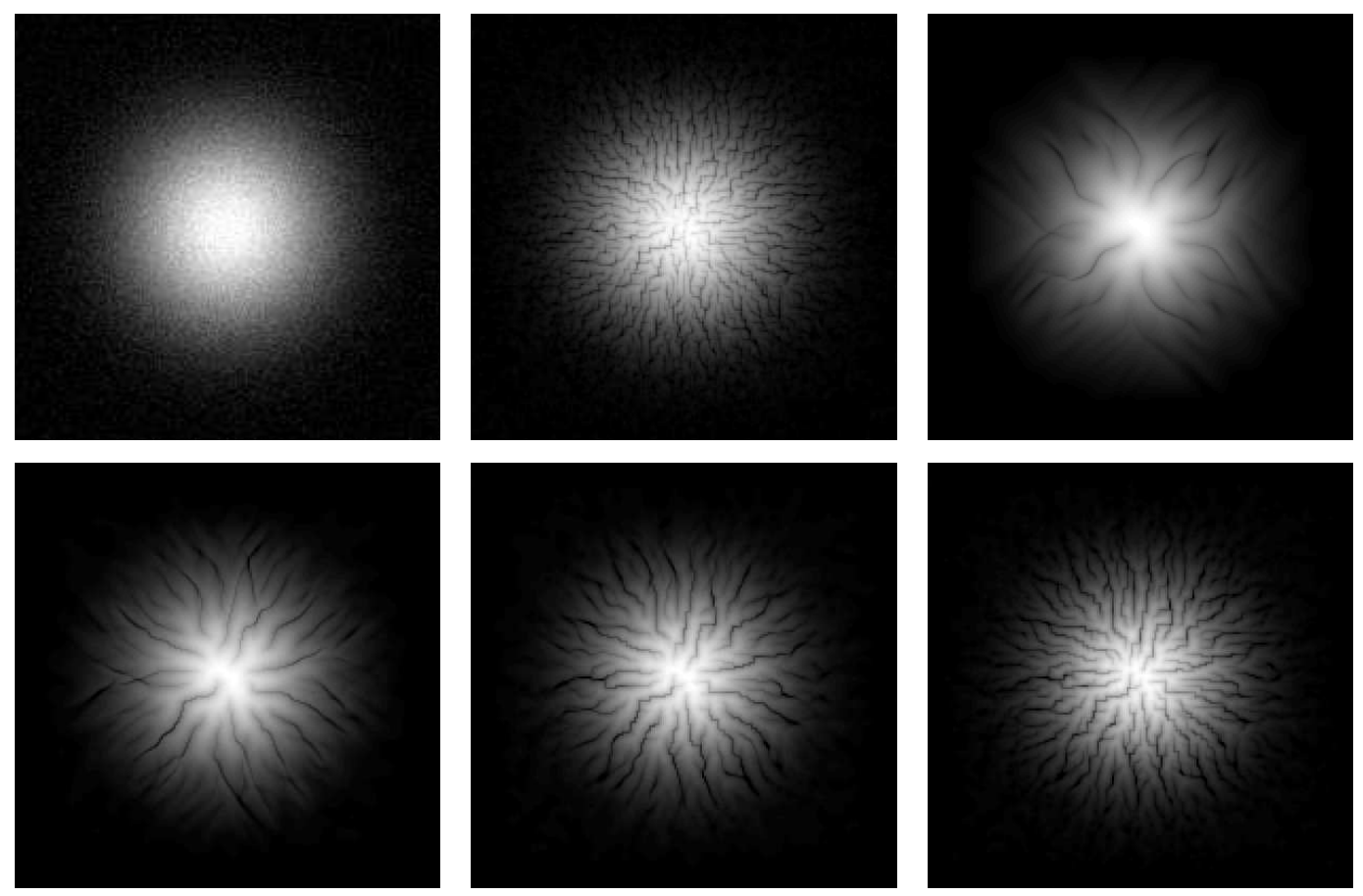

FIG. 6. Initialization with a highly channelized landscape and decreasing the rain parameter gives a final landscape qualitatively similar to that obtained by running evolution with the smaller rain parameter for the entire simulation. Top Left: Initial $201 \times 201$ landscape, a Gaussian with $N\left(0,(10 / 255)^{2}\right)$ noise added. Top middle: $r=200, e=3, c=1$, $s=1$, with a stopping condition of $20 \%$ landscape removed. Top right and bottom row: simulations with the top middle landscape as initial condition, and final condition after another $20 \%$ removed, with parameters $e=3, c=1, s=1$, variable $r$. Top right: $r=25$, Bottom row: $r=50, r=100, r=200$.

and 6. But when the new parameters support a larger channel spacing, the landscape, starting from the state represented in the second image, reverts to the lower level of channelization given by the new parameters, as apparent in results 3 to 5 , obtained by continuing the evolution with less rain.

Sedimentation fronts are observed in Nature, when rivers flow into a lake or into the ocean, depositing sediments eroded upstream that form an advancing front. Such advancing fronts are apparent in the profile plots of Figure 7. The figure is self-explanatory.

These first numerical trials of the model (4.1), (4.2) and (4.3) will now be applied to DEMs. In these images of topographical or geological surfaces, the grey level is proportional to the elevation from ocean level. Terrain elevations are obtained through a combination of techniques including remote sensing (radar, stereovision), or survey data. There are several challenges with preparing DEM data. These include sampling density and interpolation, landform variations causing distortion, and the roughness of the terrain. Furthermore, their resolution is limited by the Nyquist limits, which creates many ambiguities about the river network.

One particular problem is the introduction of small pits, which are generally acknowledged to be spurious $[30,44,48]$. Another problem is that single pixels or small patches can often erroneously block rivers $[36,40]$. Thus, pit removal and river network restoration are two important problems in 

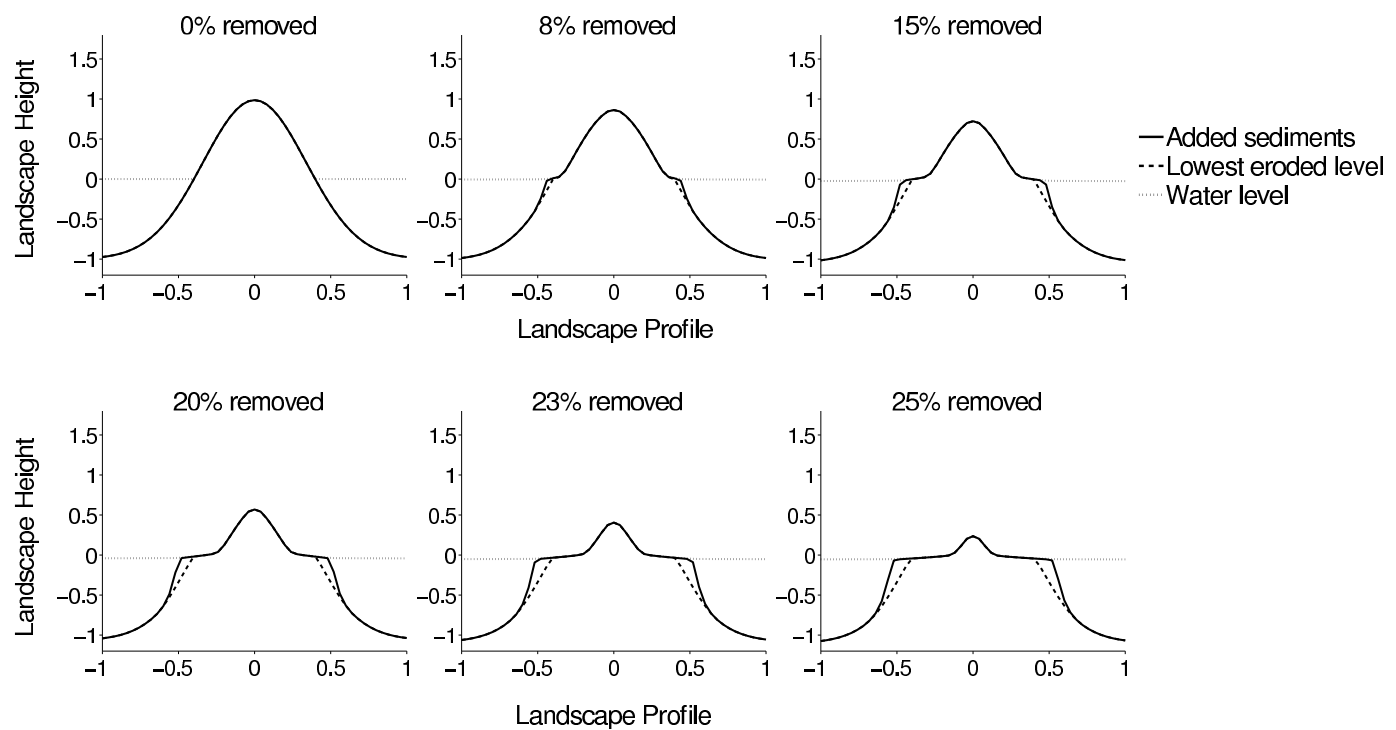

FIG. 7. Evolution profiles of a $51 \times 51$ Gaussian landscape without added noise. The initial landscape has been lowered, so that the Dirichlet "ocean" condition occurs at half the initial landscape height. Profiles in time are shown with various amounts of landscape removed. Units shown are nondimensionalized.

improving DEM quality. Needless to be said, the river network is not observable as such in the DEM and must reconstructed from it. These problems are extensively discussed and numerically efficient solutions have been proposed in the above mentioned works, and in Liu et al. [37], Planchon and Darboux [48], Soille and Gratin [61], Wang and Liu [68], Freeman [15], Tarboton [63], Seibert and McGlynn [57], Fairfield and Leymarie [12], Jenson and Domingue [30]. These numerical models are not based on PDE's, and do not simulate a landscape evolution. Their main object is the water evolution (filling in of lakes, and determination of the water flow direction) and the identification of basins.

In our presented numerical results on DEM landscape evolution, river network reconstruction and basin expansion can actually be obtained as an interesting side product of a simulation of the evolution itself. River networks that do not appear clearly in the initial DEM can be reconstructed plausibly by evolving the landscape. This ability results from an erosion model that tends to reconnect rivers with the same orientation that have been broken by a single or a few pixels. Secondly, basins (the areas downstream of river networks) expand as higher elevation areas are eroded, expanding down-slope.

The numerical examples on actual DEM's are somewhat inaccurate near the boundary, as Dirichlet boundary conditions have been used, while much of the boundary of the DEM is above a 0 landscape level. Thus, there are instabilities near the boundary that result in spurious river formation. The evolution is instead coherent in basins that happen to be entirely included in the computational domain.

The experiment of Figure 8 shows that the river network can be extracted clearly, as long as the landscape does not evolve too far away from the initial condition. For longer evolution, the river network is more detailed, but may be more inaccurate if the chosen parameters do not fit the 

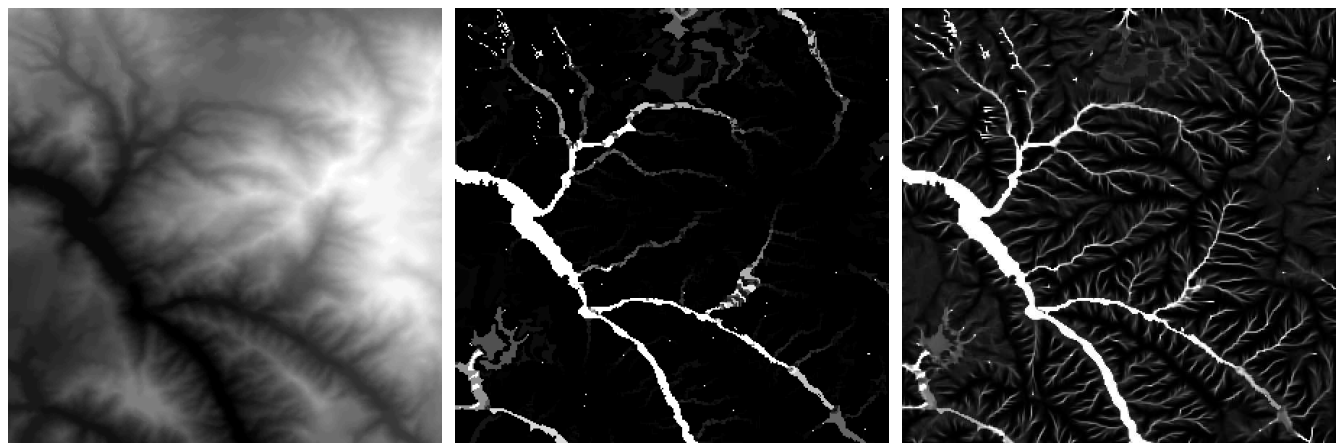

FIG. 8. River network extraction. The water level at intermediate states gives a good approximation of the river network of the DEM for a small number of iterations. Parameters: $r=5, e=1, c=1, s=0$. Left: the original DEM. Middle: the original steady state level of water. Right: the water level after $20 \%$ of the landscape is removed.

landscape. It is therefore crucial to develop a methodology to identify the best parameters for a given DEM, namely those for which a stability of the morphology is observed under the three-equation system.

Finally, we observed that two different landscapes, obviously sampled at different scales, evolve in similar ways for similar parameters. Figure 9 shows the results of landscape evolution for two different initial landscapes that have been evolved with the same parameters and the same stopping condition. The simulation shows the pure effect of erosion by water only, the creep and sedimentation parameters being zero. With these values it is easily checked that the three equations model boils down to its two first equations, (4.1) and (4.2). As we pointed out in Section 4.1, this system of equation is scale invariant and therefore prone to model a fractal evolution.

\section{Conclusion and open problems}

This paper has reviewed a choice of the papers proposing to model the evolution of landscape under the combined effects of erosion, sedimentation, and landslide. The analysis has demonstrated that two of the main constitutive equations are transport conservative equations governing the water flow and sediment transport. The third equation combines the three mentioned effects. This minimal system has three state variables (water level, landscape elevation and sediment concentration in water). It therefore does not consider the existence of two distinct layers, namely regolith and bedrock. Neither does it consider effects that influence certain kinds of landscapes, such as wind on dunes, glaciers, or water infiltration. Nevertheless, an analysis of the system and of its first numerical simulation indicates a promising tractability. On the one hand, the six model parameters give enough degrees of freedom for tuning the erosion, sedimentation and transport effects. On the other hand an analysis of the parameter interaction indicates that the number of independent parameters is closer to 3 than to 6, which might permit exhaustive experimentation and the identification of the best parameters for each landscape. Such an experimentation would point to the necessary extensions, namely the phenomena that require the addition of a new state variable, or a change in the form of the nonlinearities.

A short but significant list of mathematical conjectures can be attached to such a system of equations, which shows its mathematical interest. 

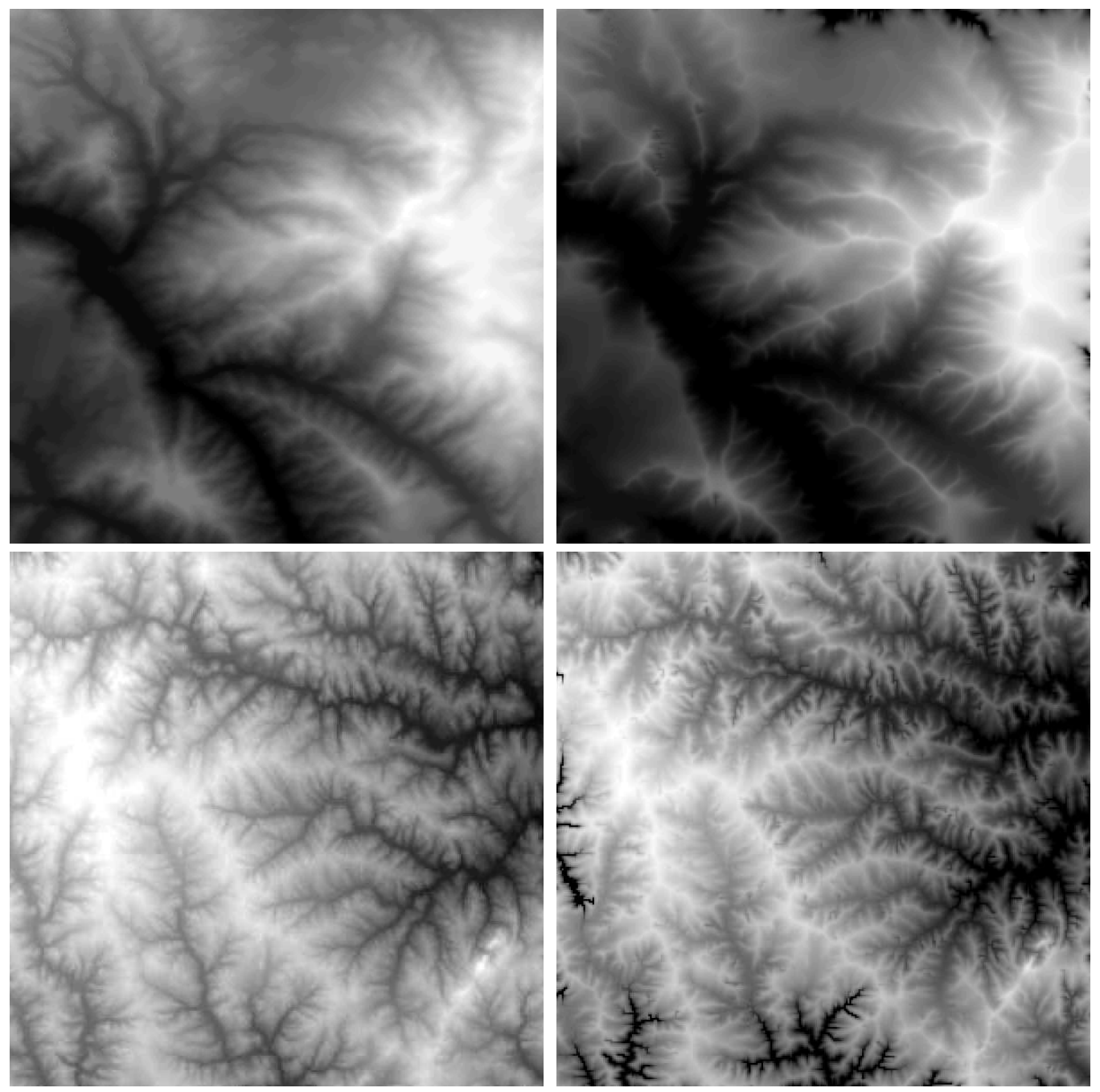

FIG. 9. A comparison of landscape evolution (with pure erosion) on two initial landscapes at different scales, but with the same parameters: $m=0.5, r=1, e=5, c=0, s=0$, with a stopping condition of $20 \%$ of the landscape removed. Top left: Initial landscape 1; Top right: Evolved landscape 1; Bottom left: Initial landscape 2; Top right: Evolved landscape 2. The evolution is similar, and indeed the model is scale invariant.

1. Local existence in time. Each of the three equations (4.1), (4.2), (4.3) seems well-posed as an evolution equation on $\theta, z$ and $\rho$, respectively, if the two other unknowns are taken as data; is it possible to show (for instance by a Schauder fixed point method) local existence of a solution to the whole system, under possible regularity assumptions on the initial data?

2. Global existence in time. Numerical simulations suggest that this local existence result could be made global for initial Lipschitz landscapes, and that the landscape remains Lipschitz continuous, under the condition that rain $r_{1}>r(t)>r_{0}$ is constant or at least bounded between 
two values, even if the $c$ creep constant is zero.

3. Non uniqueness, and instability. The possible complete divergence of two initial solutions that differ infinitesimally is also conjectured for $c=0$. On the contrary, for $c_{1}>c>c_{0}$, a unique behavior (with regularity) is also expected.

4. Regularity. It is natural to expect regularity if the creep coefficient $c$ is strictly positive; on the other hand, should we expect much less regularity in the case $c=0$ ? In particular, the Lipschitz behavior could degenerate as $t \rightarrow \infty$ into Hölder continuity, if one looks at what happens in the statical case for the landscape function in branched transportation (see Section 2.7).

5. Asymptotics as $t \rightarrow \infty$. Supposing a solution is defined for global time, what about its limit as $t \rightarrow \infty$ ? Does this limit exist (i.e. the landscape evolves to an asymptotical landscape, which seems reasonable instead of having oscillations in time) and how is it characterized? In particular we can wonder if it simply solves the corresponding statical equations (i.e., removing time derivatives from (4.1), (4.2), (4.3)) and if it is linked to the landscape function of Section 2.7. Needless to say, and as pointed out by all authors, such a system seems a priori insufficient to model any landscape in the short term. Indeed, in the short term, vegetation, climate changes, wind, animal and human factors can reshape a landscape and influence its evolution. Furthermore, our intuition of landscapes may be affected by our common interest in singularities such as recent volcanic sites, falls and glaciers. Nonetheless, on another time scale where all of these conditions have changed many times, the landscape morphology might be explained by simple equations.

\section{REFERENCES}

1. Aubert, G., \& Kornprobst, P. Mathematical problems in image processing: partial differential equations and the calculus of variations, vol. 147. Springer, 2006. Zbl1110.35001 MR2244145

2. Banavar, J. R., Colaiori, F., Flammini, A., Giacometti, A., Maritan, A., \& Rinaldo, A. Sculpting of a fractal river basin. Phys. Rev. Lett. 7823 (1997), 4522-4525.

3. Banavar, J. R., Colaiori, F., Flammini, A., Giacometti, A., Maritan, A., \& Rinaldo, A. Scaling, optimality, and landscape evolution. J. Stat. Phys. 104 1/2 (2001), 1-48. Zbl1074.86518

4. Bernot, M., CAselles, V., \& Morel, J.-M. Optimal transportation networks: models and theory. Springer-Verlag, 2009. Zb11163.90001 MR2449900

5. Birnir, B., Smith, T. R., \& Merchant, G. E. The scaling of fluvial landscapes. Comput. Geosci. 27 10 (2001), 1189-1216.

6. Brancolini, A., \& Solimini, S. On the Hölder regularity of the landscape function. Interfaces Free Bound. 132 (2011), 191-222. Zbl1220.49022 MR2813523

7. CARson, M. A., \& Kirby, M. J. Hillslope Form and Process. The University Press, 1972.

8. Chow, V. T. Open-channel hydraulics. McGraw-Hill, 1959.

9. Culling, W. E. H. Analytical theory of erosion. J. Geol. 683 (1960), 336-344.

10. Davis, W. M. The convex profile of bad-land divides. Science 508 (1892), 245.

11. Dietrich, W. E., Bellugi, D. G., Sklar, L. S., Stock, J. D., Heimsath, A. M., \& Roering, J. J. Geomorphic transport laws for predicting landscape form and dynamics. Prediction in Geomorphology, Geophys. Monogr. Ser. 135 (2003), 103-132.

12. FAirfield, J., \& Leymarie, P. Drainage networks from grid digital elevation models. Water Resour. Res. 275 (1991), 709-717.

13. Fernandes, N. F., \& Dietrich, W. E. Hillslope evolution by diffusive processes: The timescale for equilibrium adjustments. Water Resour. Res. 336 (1997), 1307-1318.

14. Flint, J. J. Stream gradient as a function of order, magnitude, and discharge. Water Resour. Res. 105 (1974), 969-973. 
15. Freeman, T. G. Calculating catchment area with divergent flow based on a regular grid. Comput. Geosci. 173 (1991), 413 - 422.

16. Gauckler, P. Etudes Théoriques et Pratiques sur l'Ecoulement et le Mouvement des Eaux. C. R. Acad. Sci. Paris 64 (1867), 818-822.

17. Gilbert, G. K. The convexity of hilltops. J. Geol. 174 (1909), 344-350.

18. Gilbert, G. K., \& Dutton, C. E. Report on the Geology of the Henry Mountains. Govt. print. off., 1877.

19. GRAF, W. H. Hydraulics of sediment transport. Water Resources Publication, 1984.

20. HARBOR, D. J. Landscape evolution at the margin of the basin and range. Geology 2512 (1997), 1111 1114.

21. Hilley, G. E., \& Arrowsmith, J. R. Geomorphic response to uplift along the Dragon's Back pressure ridge, Carrizo Plain, California. Geology 365 (2008), 367-370.

22. Horton, R. E. Erosional development of streams and their drainage basins; hydrophysical approach to quantitative morphology. Geol. Soc. Am. Bull. 563 (1945), 275-370.

23. Hovius, N. Regular spacing of drainage outlets from linear mountain belts. Basin Res. 81 (1996), 29-44.

24. Howard, A. D. Theoretical model of optimal drainage networks. Water Resour. Res. 269 (1990), 2107-2117.

25. HowARD, A. D. A detachment-limited model of drainage basin evolution. Water Resour. Res. 307 (1994), 2261-2285.

26. Howard, A. D., Dietrich, W. E., \& Seidl, M. A. Modeling fluvial erosion on regional to continental scales. J. Geophys. Res. 99 B7 (1994), 13971-13986.

27. Howard, A. D., \& Kerby, G. Channel changes in badlands. Geol. Soc. Am. Bull. 946 (1983), 739-752.

28. IjJasz-Vasquez, E., Bras, R. L., Rodriguez-Iturbe, I., Rigon, R., \& Rinaldo, A. Are river basins optimal channel networks? Adv. Water Resour. 16 (1993), 69-79.

29. IZUMI, N., \& PARKER, G. Inception of channelization and drainage basin formation: upstream-driven theory. J. Fluid Mech. 283 (1995), 341-363. Zb10865.76026

30. Jenson, S. K., \& Domingue, J. O. Extracting topographic structure from digital elevation data for geographic information system analysis. Photogramm. Eng. Remote Sens. 5411 (1988), 1593-1600.

31. KIRKBY, M. J. Hillslope process-response models based on the continuity equation. I. Brit. Geogr. Special Publication 3 (1971), 15-30.

32. KIRKBY, M. J. Modelling some influences of soil erosion, landslides and valley gradient on drainage density and hollow development. Catena, 1987.

33. LEOPOLD, L. B., \& MADDOCK, T. The hydraulic geometry of stream channels and some physiographic implications. Geol. Surv. Prof. Pap. U.S. 252 (1953).

34. LeOPOlD, L. B., \& Miller, J. P. Ephemeral streams: hydraulic factors and their relation to the drainage net. Geol. Surv. Prof. Pap. U.S. 282 (1956), 1-36.

35. Leopold, L. B., Wolman, M. G., \& Miller, J. P. Fluvial processes in geomorphology. William Freeman, San Francisco, 1964.

36. Lindsay, J. B., \& CREED, I. F. Removal of artifact depressions from digital elevation models: towards a minimum impact approach. Hydrol. Process. 1916 (2005), 3113-3126.

37. LIU, Y.-H., ZHANG, W.-C., \& XU, J.-W. Another fast and simple DEM depression-filling algorithm based on priority queue structure. Atmos. Ocean Sci. Lett. 24 (2009), 214-219.

38. LOEWENHERZ, D. S. Stability and the initiation of channelized surface drainage: a reassessment of the short wavelength limit. J. Geophys. Res. 96 B5 (1991), 8453-8464.

39. Mallet, J. L. Space-time mathematical framework for sedimentary geology. Math. Geol. 361 (2004), 1-32. Zbl1042.86514 MR2040991

40. Martz, L. W., \& GARBrecht, J. An outlet breaching algorithm for the treatment of closed depressions 
in a raster DEM. Comput. Geosci. 257 (1999), 835 - 844.

41. Moglen, G. E., Eltahir, E. A. B., \& Bras, R. L. On the sensitivity of drainage density to climate change. Water Resour. Res. 344 (1998), 855-862.

42. Montgomery, D. R., \& Dietrich, W. E. Source areas, drainage density, and channel initiation. Water Resour. Res. 258 (1989), 1907-1918.

43. Niemann, J. D., Gasparini, N. M., Tucker, G. E., \& Bras, R. L. A quantitative evaluation of Playfair's law and its use in testing long-term stream erosion models. Earth Surf. Proc. Land. 26 (2001), 1317-1332.

44. O'Callaghan, J. F., \& MARK, D. M. The extraction of drainage networks from digital elevation data. Comput. Vision Graph. 283 (1984), 323 - 344.

45. Passalacqua, P., Do Trung, T., Foufoula-Georgiou, E., Sapiro, G., \& Dietrich, W. E. A geometric framework for channel network extraction from LIDAR: Nonlinear diffusion and geodesic paths. J. Geophys. Res. 115 F1 (2010), F01002.

46. Perron, J. T., Dietrich, W. E., \& Kirchner, J. W. Controls on the spacing of first-order valleys. $J$. Geophys. Res. 113 F4 (2008), F04016.

47. Perron, J. T., Kirchner, J. W., \& Dietrich, W. E. Formation of evenly spaced ridges and valleys. Nature 4607254 (2009), 502-505.

48. Planchon, O., \& Darboux, F. A fast, simple and versatile algorithm to fill the depressions of digital elevation models. Catena 46 2ñ3 (2002), 159 - 176.

49. Rigon, R., Rinaldo, A., Rodriguez-Iturbe, I., IjJasz-Vasquez, E., \& Bras, R. L. Optimal channel networks: a framework for the study of river basin morphology. Water Resour. Res. 296 (1993), 1635-1646.

50. Rinaldo, A., Dietrich, W. E., Rigon, R., Vogel, G. K., \& Rodrlguez-Lturbe, I. Geomorphological signatures of varying climate. Nature 3746523 (1995), 632-635.

51. Robiins, J. A., \& Edgington, D. Determination of recent sedimentation rates in Lake Michigan using Pb-210 and Cs-137. Geochim. Cosmochim. Ac. 393 (1975), 285 - 304.

52. RodrigueZ-Iturbe, I., \& Rinaldo, A. Fractal river basins: chance and self-organization. Cambridge Univ Pr, 2001.

53. Rodriguez-Iturbe, I., Rinaldo, A., Rigon, R., Bras, R. L., IJjasz-Vasquez, E., \& Marani, A. Fractal structures as least energy dissipation patterns: the case of river networks. Geophys. Res. Lett. 5 (1992), 2854-2860.

54. RoERING, J. J. How well can hillslope evolution models "explain" topography? Simulating soil transport and production with high-resolution topographic data. Geol. Soc. Am. Bull. 120 (2008), 1248-1262.

55. Roering, J. J., Kirchner, J. W., \& Dietrich, W. E. Evidence for nonlinear, diffusive sediment transport on hillslopes and implications for landscape morphology. Water Resour. Res. 353 (1999), 853870.

56. Santambrogio, F. Optimal channel networks, landscape function and branched transport. Interfaces Free Bound. 91 (2007), 149-169.

57. Seibert, J., \& McGlynn, B. L. A new triangular multiple flow direction algorithm for computing upslope areas from gridded digital elevation models. Water Resour. Res. 434 (2007), W04501.

58. Seidl, M. A., \& Dietrich, W. E., The problem of channel erosion into bedrock. Catena suppl. 23 (1993), 101-124.

59. Simpson, G., \& SChlunegger, F. Topographic evolution and morphology of surfaces evolving in response to coupled fluvial and hillslope sediment transport. J. Geophys. Res. 108 B6 (2003), 2300.

60. Smith, T. R., \& Bretherton, F. P. Stability and the conservation of mass in drainage basin evolution. Water Resour. Res 86 (1972), 1506-1529.

61. Soille, P., \& Gratin, C. An efficient algorithm for drainage network extraction on DEMs. J. Vis. Commun. Image R. 52 (1994), 181 - 189. 
62. Sole, A., Caselles, V., Sapiro, G., \& Arandiga, F. Morse description and geometric encoding of digital elevation maps. IEEE T. Image Process. 139 (2004), 1245-1262. MR2093410

63. TARboton, D. G. A new method for the determination of flow directions and upslope areas in grid digital elevation models. Water Resour. Res. 332 (1997), 309-319.

64. Tarboton, D. G., Bras, R. L., \& Rodriguez-Iturbe, I. A physical basis for drainage density. Geomorphology 5 1-2 (1992), 59-76.

65. TUCKer, G. E., \& BRAS, R. L. Hillslope processes, drainage density, and landscape morphology. Water Resour. Res. 3410 (1998), 2751-2764.

66. Tucker, G. E., \& Slingerland, R. Drainage basin responses to climate change. Water Resour. Res. 338 (1997), 2031-2047.

67. Vallet, G. Sur une loi de conservation issue de la géologie. C. R. Math. 3378 (2003), 559-564. Zbl1038.86010 MR2017697

68. WANG, L., \& LIU, H. An efficient method for identifying and filling surface depressions in digital elevation models for hydrologic analysis and modelling. Int. J. Geogr. Inf. Sci. 202 (2006), 193-213. Zb11190.65034

69. Welsh, E., Birnir, B., \& Bertozzi, A. Shocks in the evolution of an eroding channel. Appl. Math. Res. Express 20060 (2006), 71638. Zbl1116. 35341 MR2278493

70. Willgoose, G., Bras, R. L., \& Rodriguez-Iturbe, I. A coupled channel network growth and hillslope evolution model: 2. Nondimensionalization and applications. Water Resour. Res. 277 (1991), 1685-1696.

71. XIA, Q. On landscape functions associated with transport paths. Discrete Contin. Dyn. S. 344 (April 2014), 1683-1700. Zbl1278.90053 